Document downloaded from:

http://hdl.handle.net/10251/56625

This paper must be cited as:

Pacheco Páramo, DF.; Pla, V.; Casares Giner, V.; Martínez Bauset, J. (2012). Optimal Radio Access Technology Selection on Heterogeneous Networks. Physical Communication. 5(3):253-271. doi:10.1016/j.phycom.2012.02.009.

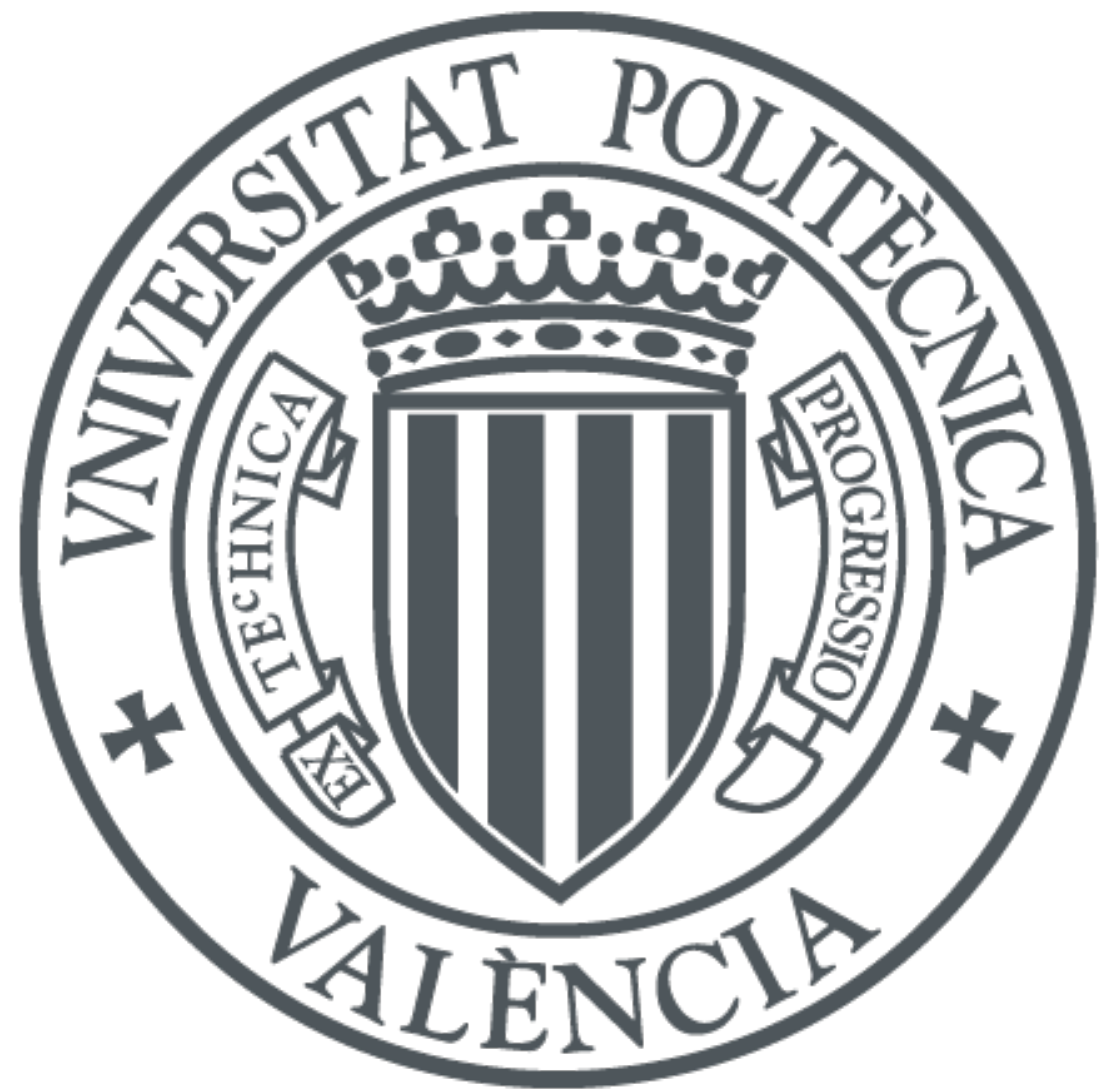

The final publication is available at

http://dx.doi.org/10.1016/j.phycom.2012.02.009

Copyright Elsevier

Additional Information 


\title{
Optimal Radio Access Technology Selection on Heterogeneous Networks
}

\author{
Diego Pacheco-Paramo, Vicent Pla, Vicente Casares-Giner and Jorge Martinez-Bauset \\ Dept. Comunicaciones, Universidad Politecnica de Valencia \\ Camino de Vera s/n, 46022, Valencia, Spain \\ Email: diepacpa@posgrado.upv.es,\{vpla,vcasares,jmartinez\}@dcom.upv.es
}

\begin{abstract}
The joint management of radio resources in heterogeneous networks is considered to raise the resource occupation efficiency. We propose joint schemes for admission control and access technology selection with vertical handoff. We determine optimal policies for a radio systems that support two multiple access techniques, TDMA and WCDMA, covering the same geographical area. In addition, the system under study also supports both streaming and elastic traffic. First, we explore different optimization criteria expressed in terms of blocking probabilities and throughput. We accomplish an exhaustive numerical analysis that allows us to characterize the optimal admission policies according to the arrival type and system load. Based on this characterization, we design heuristic policies and compare their performance to ones obtained by previously proposed schemes. This analysis is also done when constraints are added to provide QoS. Second, we also study an extension of the previous system that includes vertical handoffs, in order to evaluate their impact on the system performance. For the four types of vertical handoffs considered, we determine and characterize optimal policies according to the arrival type, system load and vertical handoff action. Since it is not feasible to calculate the optimal policies online, new heuristic policies with vertical handoff are constructed and evaluated. Finally, in order to take into into account the cost of performing vertical handoffs, a new optimization problem is formulated that relates voice and data blocking with vertical handoff costs.
\end{abstract}

Keywords: joint call admission control, vertical handoff, resource allocation, Markov decision process

\section{Introduction}

The new generation wireless systems allow the subscribers to exploit the availability of multiple radio access technologies (RATs) in the same geographical area to achieve a permanent connection with the best possible performance [1]. This coexistence of various RATs has promoted the interest in joint management of their radio resources. Common radio resource management (CRRM) has been proposed as a model to control several RATs in a coordinated way, exploiting the fact that this coordination allows an improvement in the use of the limited resources [2]. Among the CRRM functions, there is a special interest in the joint call admission control (JCAC), which in this new scenario not only defines if a call should be accepted but also in what technology, depending on user's or operator's criteria [3].

Proposals for JCAC in heterogeneous networks, such as those found in [4] and [5], show the improvement in network's stability based on a load balancing scheme.
Even though, some other advantages can be exploited from a common admission control scheme. In this article, we are interested in finding an optimal scheme for a heterogeneous network with two technologies, and where both streaming and elastic traffic are supported. Several JCAC policies have been studied in [5] for a scenario similar to ours, in terms of throughput and blocking probability. Nevertheless, it has to be considered that the best possible policy may not follow a simple scheme, since its behavior may be affected by different parameters that depend on the technologies involved or the traffic characteristics. Therefore, in this paper there is an analytical study to obtain the optimal policy and its main characteristics in relation to the parameters named before. Similar works have been done in order to define a scheme that enhance network's performance.

In [6] a Markov model is used for an heterogeneous network (WLAN and CDMA), and a linear programming method is used to solve the optimization problem. This study explicitly acknowledges the inherent 
complexity of the problem which could make it computationally intractable for large systems, and proposes reinforcement learning as a possible solution method. Although we still have that limitation, the objective of this work is to obtain the main characteristics of the optimal solutions to propose heuristic schemes that can be extrapolated to bigger systems. In [7] a genetic algorithm is used to find a common access control scheme based on a Markov model and a cost function which depends on several factors. However, the initial conditions play a crucial role in the results obtained by genetic algorithms, therefore affecting the objective of obtaining heuristic solutions that apprehend the main characteristics of the optimal solutions under different conditions.

Finally, fuzzy-based solutions are used in [8] and [9]. This solutions take different parameters from the user and the system, weights them, and make a decision into a complex online solution. On the other hand, our focus is to synthesize the characteristics of the optimal solutions for two explicit optimization functions: throughput and blocking probabilities. As other works mentioned before, a Markovian model is used, but the Markov decision process is solved based on policy iteration, a method that does not depend on the initial conditions used and always allows to find the optimal solution in a finite and usually low number of steps. Once the main characteristics of the optimal solutions are described in relation to the system and traffic characteristics, a new access control scheme is proposed and analyzed.

Acknowledging the importance that vertical handoffs have as part of the joint radio resource management functions, and based on the analysis previously done on the system, different types of vertical handoffs are introduced according to the type of the arriving service and some specific conditions of the system. Since there is an inherent complexity in vertical handoff as it is pointed out in [3], it is required that the proposed schemes do not boost it. On the other hand, most vertical handoff schemes like those in [14],[15], privilege user preferences, and we consider that the operator's point of view is also important. The last part of the article focuses on the cost of vertical handoffs. In previous studies like [16], it is defined a monetary cost for this function so users can use it as a parameter to decide which technology is more suitable. On our work, we relate this cost to those associated to the blocking of voice and data sessions in order to understand when it is useful.

The rest of the paper is structured as follows. In Section 2 we describe the Markov model according to the characteristics of the system. The solution method is described in Section 3. In Section 4 the results of the opti- mization are shown, and analyzed for a static scenario. The optimal solutions for various traffic values are studied in Section 5. In Section 6, we add QoS restrictions into optimization and analyze its results. Section 7 introduces vertical handoff in the action set, and new optimal policies are found in order to obtain the main characteristics of the optimal solutions. New heuristic solutions are described and evaluated in different scenarios and simulations are performed in order to validate analytic results. In Section 8 we study the cost of performing a single vertical handoff to understand its impact in relation to performing data and voice blocking. Finally, Section 9 concludes the work.

\section{System description and Markov model}

The system of our interest uses two multiple access techniques, TDMA and WCDMA. Their characteristics allow us to model technologies such as GSM and UMTS. However,the proposed model and analysis can be expanded to cover other multiple access techniques like OFDMA, which has been defined to support technologies like WiMAX and LTE. Additionally, both access techniques provide voice and data services in the same area as it was proposed in [10]. As a call arrives to the system, a decision has to be made about if it is served in one or another technology. Initially, we consider that a decision is made once a session starts and will be held until is served, i.e. there are no vertical handoffs.

In order to obtain an analytically tractable model, we assume that voice (data) call (session) arrivals follow a Poisson process with rate $\lambda_{v}\left(\lambda_{d}\right)$. We also assume that the service time for voice calls is exponentially distributed with mean $1 / \mu_{v}$. On the other hand, as data sessions generate elastic traffic, their sojourn time will depend on the available resources. The size of the flows generated by the data sessions is exponentially distributed with mean $\sigma$ (in bits). If $B R_{d}$ is the data bit rate experienced for a given user, then the service time will be exponentially distributed with mean $1 / \mu_{d}=\sigma / B R_{d}$. Clearly, $B R_{d}$ might depend on the system state as discussed later.

\subsection{State space}

The system is modeled as a 4 dimensional continuous time Markov chain (CTMC), with state represented by the state vector $s=\left(s_{1}, s_{2}, s_{3}, s_{4}\right)$ where $s_{1}$ represents the number of ongoing voice sessions on TDMA, $s_{2}$ the data sessions on TDMA, $s_{3}$ the voice sessions on WCDMA and $s_{4}$ the data sessions on WCDMA. 
We define $C$ as the fixed number of channels per slot in TDMA. A voice session will always use a whole channel, so there can only be $C$ simultaneous voice sessions on this technology. On the other hand, data sessions can share a channel when TDMA is at full capacity, in such a way that $n_{c}$ data sessions can be served per channel. This means that we can have a maximum of $C \cdot n_{c}$ simultaneous data sessions in TDMA. According to this, the first condition that a state must fulfill to be feasible is given by

$$
s_{1} \cdot n_{c}+s_{2} \leq n_{c} \cdot C .
$$

The capacity on WCDMA is defined by

$$
s_{3}\left(\frac{W / B R_{w, v}}{\left(E_{b} / N_{0}\right)_{v}}+1\right)^{-1}+s_{4}\left(\frac{W / B R_{w, d}}{\left(E_{b} / N_{0}\right)_{d}}+1\right)^{-1} \leq \eta_{u l},
$$

where $W$ is the chip rate, $B R_{w, x}$ is the bit rate used for transmitting service $x$ in WCDMA, $\left(E_{b} / N_{0}\right)_{x}$ is the bit energy to noise density required for service $x$, and $\eta_{u l}$ is the uplink cell load factor. This is the same expression used in [11].

Considering that each technology has independent resources, the feasible combination of data and voice users can be determined for each technology separately. We define $S$ as the set of feasible states, that is all the state vectors $s$ that fulfill the conditions defined in (1) and (2).

\subsection{System metrics}

After the CTMC is constructed, that is we have defined the state space and the transition rates, we obtain different system metrics using the steady state probabilities. In particular, we are interested in the voice blocking probability, the data blocking probability, and the total throughput. The total blocking probability refers to the probability that the system is in a state where all calls are blocked, independent of the service it requires. In the same way the voice (data) blocking probability refers to the probability of being in those states where voice (data) calls are blocked. To calculate the throughput we have to consider that the bit rate is independent for each service and technology and that data sessions in TDMA can share a channel, which is reflected in the $\min \left(C-s_{1}, s_{2}\right)$ factor of the following equation:

$$
\begin{aligned}
T h= & \sum_{s \in S} P(s)\left(s_{1} B R_{t, v}+s_{3} B R_{w, v}\right. \\
& \left.+\min \left(C-s_{1}, s_{2}\right) B R_{t, d}+s_{4} B R_{w, d}\right),
\end{aligned}
$$

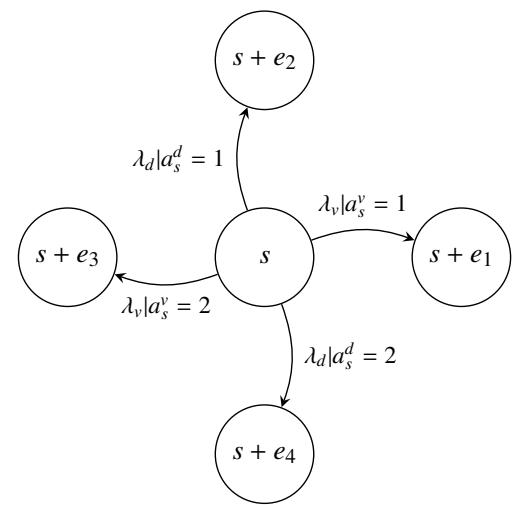

Figure 1: Transitions for voice and data arrivals.

Table 1: Set of possible actions A

\begin{tabular}{|c|c|}
\hline value & action \\
\hline 0 & Block call \\
1 & Send call to TDMA \\
2 & Send call to WCDMA \\
\hline
\end{tabular}

where $P(s)$ is the steady state probability of being in state $s$ and $B R_{x, y}$ is the bit rate used for transmitting service $y$ (voice or data) in technology $x$ (TDMA or WCDMA).

\section{Optimization problem}

For each possible state defined in the last section, a decision must be made whether an arriving call should be admitted and given the case, the appropriate technology according to the service, i.e. voice or data. In a Markov decision process (MDP), a policy $\pi$ defines which actions $a=\left(a_{s}^{v}, a_{s}^{d}\right)$ should be taken at each state $s$ for voice $a_{s}^{v}$ and data $a_{s}^{d}$ arrivals. It should be clear that decision epochs occur only at arrivals. The main objective is to find among the possible policies the one that optimizes a chosen function.

In this system, the set of actions $A$ defines the possible values for $a_{s}^{v}$ and $a_{s}^{d}$, and it is defined in Table 1 . Figure 1 shows the transitions from state $s$ for arrivals conditioned by $a_{s}^{v}$ and $a_{s}^{d}$, where $e_{i}$ is a 4 dimensional vector of zeros with a 1 on the $\mathrm{i}$-th position. The transitions from state $s$ for departures are shown in Figure 2.

We define now three different admission schemes and set the values taken by $a_{s}^{x}$ accordingly, where $x$ could be voice or data. Let $A C_{T D M A}$ and $A C_{W C D M A}$ be the available free capacity in TDMA and WCDMA, 


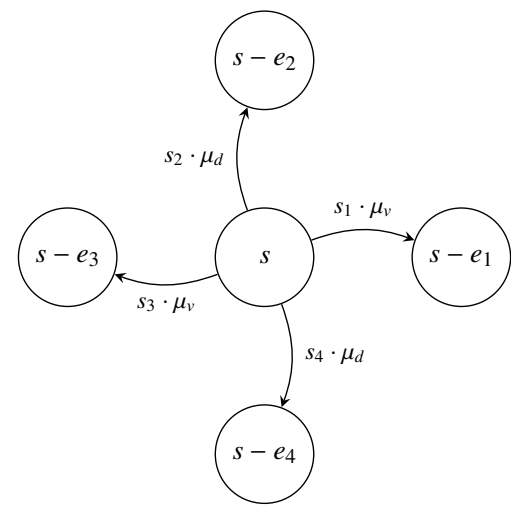

Figure 2: Transitions for voice and data departures.

respectively.

Scheme $i$ : Calls are sent to TDMA (if possible)

$$
a_{s}^{x}= \begin{cases}1 & \text { if } A C_{T D M A}>0 \\ 2 & \text { if } A C_{T D M A}=0 \text { and } A C_{W C D M A}>0 \\ 0 & \text { if } A C_{T D M A}=A C_{W C D M A}=0\end{cases}
$$

Scheme ii: Calls are sent to WCDMA (if possible)

$$
a_{s}^{x}= \begin{cases}2 & \text { if } A C_{W C D M A}>0 \\ 1 & \text { if } A C_{W C D M A}=0 \text { and } A C_{T D M A}>0 \\ 0 & \text { if } A C_{T D M A}=A C_{W C D M A}=0\end{cases}
$$

Scheme iii: Calls are sent to the technology with lower occupation

$$
a_{s}^{x}= \begin{cases}1 & \text { if } A C_{T D M A}>A C_{W C D M A} \\ 2 & \text { if } A C_{W C D M A}>A C_{T D M A} \\ \text { random } & \text { if } A C_{W C D M A}=A C_{T D M A}>0 \\ 0 & \text { if } A C_{W C D M A}=A C_{T D M A}=0\end{cases}
$$

Using these schemes we define three policies:

\begin{tabular}{|c|c|c|}
\hline Policy & Scheme for voice & Scheme for data \\
\hline 1 & i & ii \\
\hline 2 & ii & i \\
\hline 3 & iii & iii \\
\hline
\end{tabular}

It should be noted that Policies \#1 and \#2 exploit the hypothesis that each technology is more appropriate for a specific service, which is not the case of Policy \#3 that focuses on improving radio resource utilization.

\subsection{Cost function}

Considering that the state space of the MDP is defined by (1) and (2), and the possible set of actions and the transition rates associated to them are defined in Table 1 and Figures 1 and 2, it is necessary to define the optimization functions and the cost/reward associated to them.

Since our interest relies on data and voice blocking probabilities, as well as the total throughput, we have defined two different objective functions. The first one, is the weighted sum of the voice and data blocking probabilites $\left(B P_{v}\right.$ and $\left.B P_{d}\right)$,

$$
F_{B P}=B P_{v} \cdot \alpha+B P_{d} \cdot(1-\alpha) .
$$

The parameter $\alpha, 0 \leq \alpha \leq 1$, is the one responsible for giving more or less weight to each blocking probability. When $\alpha$ is closer to 0 , minimizing the objective function will have a bigger impact on data blocking probability than on voice blocking probability, and the opposite will happen when $\alpha$ is closer to 1 .Thus, $\alpha$ relates the way in which the blocking probabilities will be minimized. The cost function associated to the objective function for each feasible state $s$ is

$$
\operatorname{cost}(s)=1-\left(\alpha \cdot F_{v}\left(a_{s}\right)+(1-\alpha) \cdot F_{d}\left(a_{s}\right)\right),
$$

where $F_{x}\left(a_{s}\right)=1$ if $a_{s}$ is 1 or 2 , and 0 otherwise, being $x$ the service.

The second objective function is the aggregated throughput, so in that case we try to maximize the value defined by (3). The reward for each state $s$ is

$$
\begin{aligned}
\operatorname{cost}(s) & =s_{1} B R_{t, v}+s_{3} B R_{w, v} \\
+ & \min \left(C-s_{1}, s_{2}\right) B R_{t, d}+s_{4} B R_{w, d} .
\end{aligned}
$$

Therefore, the reward associated to each state when maximizing the throughput does not depend on the actions taken on that state. It must be noted that we use the term cost for notational purposes, even when a reward is expected from throughput.

\subsection{Solution method}

The method used to find the optimal policy $\pi_{o p t}$ is policy iteration [12]. This method can search among the finite group of possible policies for the MDP and find the optimal in a finite number of steps. The relative values $V$ allow to relate the cost obtained in the actual state with costs expected from future actions, and are found using the next equation:

$$
\mathbf{c}^{\pi}-c_{\pi} \cdot e+V_{\pi} R_{\pi}^{T}=0 .
$$


Table 2: Policy iteration algorithm.

\begin{tabular}{|c|c|}
\hline Step 1. & Choose an arbitrary policy $\pi_{i}$ \\
\hline Step 2. & $\begin{array}{l}\text { Calculate relative values } V_{\pi_{i}} \text { and the mean } \\
\operatorname{cost} \text { (revenue) } c_{\pi_{i}} \text { for the initial policy } \pi_{i} \\
\text { using expression } 7\end{array}$ \\
\hline Step 3. & $\begin{array}{l}\text { Find action } a \text { for each state } s \text { that } \\
\text { minimize (maximize) the expression in } 8 \text {. } \\
\text { The resulting policy is called } \pi_{f} \text {. }\end{array}$ \\
\hline Step 4. & $\begin{array}{l}\text { If the resulting policy } \pi_{f} \text { differs from the } \\
\text { initial policy } \pi_{i} \text {, go back to step } 2 \text {, using } \\
\pi_{f} \text { as the initial policy. If not, } \pi_{f} \text { is the } \\
\text { optimal policy. }\end{array}$ \\
\hline
\end{tabular}

The $\mathbf{c}^{\pi}$ in the previous expression is the vector of costs associated to being in each state, $R_{\pi}$ is the transition matrix, and $c_{\pi}$ is the value of the objective function for policy $\pi$.

Once $V$ and $c_{\pi}$ are found using (7), it is possible to find the action on each state that will minimize the objective function using the next expression:

$$
\min _{a}\left\{c_{s}^{a}-c_{\pi}+\sum_{s \neq u} r_{s, u}^{a}\left(v_{\pi}^{u}-v_{\pi}^{s}\right)\right\}
$$

where $v_{\pi}^{u}$ and $v_{\pi}^{s}$ are the relative values for states $u$ and $s$ respectively when the policy $\pi$ is used, $c_{s}^{a}$ is the cost associated to state $s$ when action $a$ is taken, $r_{s, u}^{a}$ is the transition rate from state $s$ to state $u$, when the action for state $s$ is $a$. The set of actions will define a new policy and the process is repeated until the optimal policy is found. In Table 2 the policy iteration algorithm is shown. It is important to recall that the optimal policy will be found no matter what initial policy is used. However, the number of iterations could change.

\section{Optimal Policy Analysis: Static Scenario}

The parameters of the system are defined in Table 3. As it can be seen, bit rates are independent of the technology used, and are higher for data than for voice. Also, the $\left(E_{b} / N_{0}\right)$ required for both services in WCDMA are the same, and along with the parameters $C$ and $n_{c}$ from TDMA, their values are chosen in order to mantain a similar capacity on both technologies while at the same time keeping the optimization problem computationally tractable. The maximum voice and data capacities are of 4 and 8 users in TDMA and of 13 and 4 users in WCDMA. The objective of this section is to explore the optimal policy when the two optimization
Table 3: Initial Scenario for Policy Iteration.

\begin{tabular}{|c|c|}
\hline WCDMA & TDMA \\
\hline$W=3.84 \mathrm{Mcps}$ & $C=4$ \\
$\left(E_{b} / N_{0}\right)_{v}=14 \mathrm{~dB}$ & $n_{c}=2$ \\
$\left(E_{b} / N_{0}\right)_{d}=14 \mathrm{~dB}$ & $B R_{t, v}=12.2 \mathrm{kbps}$ \\
$B R_{w, v}=12.2 \mathrm{kbps}$ & $B R_{t, d}=44.8 \mathrm{kbps}$ \\
$B R_{w, d}=44.8 \mathrm{kbps}$ & \\
$\eta_{u l}=1$ & \\
\hline \hline Clients & \\
\hline$\lambda_{v}=0.025$ & \\
$\lambda_{d}=0.134$ & \\
$\mu_{v}=0.0083$ \\
$\sigma=1 \mathrm{Mb}$ \\
\hline
\end{tabular}

functions are used: blocking probability and throughput.

\subsection{Blocking function optimization}

Unless otherwise stated, throughout the document we use a value of $\alpha=0.5$. That is, the objective function will be defined by $F_{B P}(\pi)=0.5 \cdot B P_{v}+0.5 \cdot B P_{d}$. Fig. 3 shows the value of the objective function for every iteration until the optimal policy is achieved and results are compared with those obtained with the fixed policies described in Section 3. We can summarize the main characteristics of the optimal policy as follows:

\begin{tabular}{|c|l|}
\hline Service & Action \\
\hline Voice & scheme ii \\
\hline Data & $\begin{array}{l}\bullet \text { if there is no channel sharing } \\
\text { on TDMA: scheme } i \\
\bullet \text { if there is channel sharing } \\
\text { on TDMA: } \cong \text { scheme iii }\end{array}$ \\
\hline
\end{tabular}

The initial policy, Policy \#2, has the closest performance to the optimal policy because they both send voice calls to WCDMA (scheme ii). The decisions for data calls depend on channel sharing since this reduces the transmission rate, which raises the residence time and thus the blocking probability. Therefore, channel sharing produces a policy very similar to scheme iii where resources are used according to occupation.

Only $22.3 \%$ of $a_{s}^{d}$ from Policy \#2 differ from the optimal, and there is only a $6.1 \%$ probability for these actions to occur. However, results differ considerably. The cost for the optimal policy is $34.78 \%$ of the one obtained with Policy \#2, and of course similar results are found 


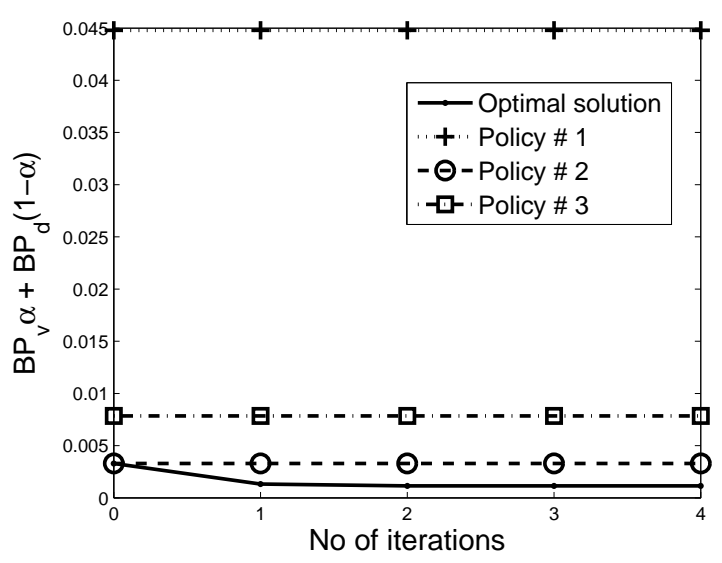

Figure 3: Blocking probability function optimization.

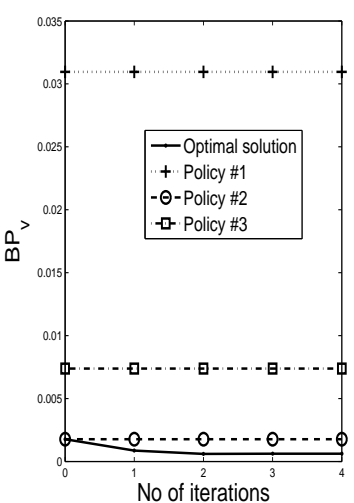

(a) VoiceBlockingProbability.

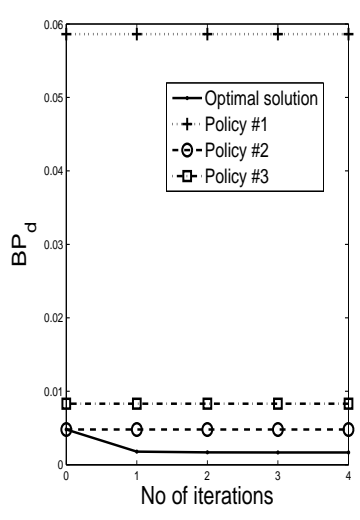

(b) DataBlockingProbability.
Figure 4: Blocking Probabilities.

on $B P_{v}$ and $B P_{d}$, as it is shown in Figs. 4(a) and 4(b). This improvement also added 462 bps to the throughput obtained with Policy \#2.

\subsection{Throughput optimization}

The characteristics of the optimal policy for throughput optimization, can be summarized as:

\begin{tabular}{|c|l|}
\hline Service & Action \\
\hline Voice & scheme ii with blocking \\
\hline Data & $\begin{array}{l}\bullet \text { if there is no channel sharing } \\
\text { on TDMA: scheme } i \\
\bullet \text { if there is channel sharing } \\
\text { on TDMA: } \cong \text { scheme } \text { iii }\end{array}$ \\
\hline
\end{tabular}

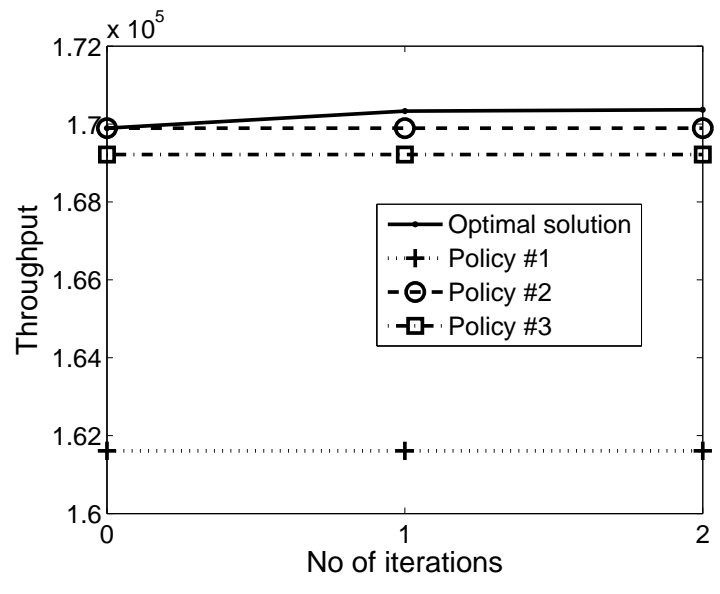

Figure 5: Throughput optimization.

Although voice calls are sent to WCDMA (scheme ii), there may be blocking when there is available capacity on TDMA. This happens in order to save capacity for data calls, which contribute more to the total throughput. In the optimal policy, nearly $25 \%$ of the states where no more capacity is available on WCDMA, even if there is capacity in TDMA, voice calls are blocked. Also, since channel sharing on TDMA directly reduces the total throughput, a policy similar to scheme iii is used for data calls.

Figure 5 shows the throughput until the optimal policy is reached. After two iterations, the throughput raises to $170.366 \mathrm{kbps}$, a gain of $474 \mathrm{bps}$ over Policy $\# 2$. The policy obtained with the first iteration is very similar to the optimal, since the second iteration only improves the total throughput in $35.8 \mathrm{bps}$, about $8 \%$ of the 438.9 bps gained before. Therefore, it is possible to use this sub-optimal solution in order to solve larger systems, in a similar approach as it was used in [13]. An interesting fact of throughput optimization, is that it also improves $B P_{v}$ and $B P_{d}$. Since these parameters have very low values in the initial policy the improvement is small, going from $0.17 \%$ to $0.079 \%$ for $B P_{v}$ and from $0.48 \%$ to $0.15 \%$ for $B P_{d}$, but it shows their correlation with the total throughput. Actually, the $B P_{d}$ is lower than the one obtained optimizing the blocking function $(0.167 \%)$, indicating the importance of accepting data calls for the improvement of the total throughput.

\section{Optimal Policy Analysis: Parameter Variation}

In this section we study the impact of traffic values over the optimal policy and based on the analysis of dynamic behaviour we define a heuristic policy. 


\subsection{Voice users arrival rate variation}

Although they are not identical, the main characteristics of optimal policies for both optimization functions as $\lambda_{v}$ grows can be summarized as:

\begin{tabular}{|l|l|l|}
\hline Service & Lower $\lambda_{v}$ & Higher $\lambda_{v}$ \\
\hline Voice & scheme ii & $\begin{array}{l}\text { scheme ii with } \\
\text { blocking }\end{array}$ \\
\hline Data & $\begin{array}{l}\text { If there is no } \\
\text { channel sharing } \\
\text { on TDMA: } \\
\text { scheme } \mathrm{i}\end{array}$ & $\begin{array}{l}\text { If there is no } \\
\text { channel sharing } \\
\text { on TDMA: } \\
\text { scheme } i\end{array}$ \\
\cline { 2 - 3 } & $\begin{array}{l}\text { if there is } \\
\text { channel sharing } \\
\text { on TDMA: } \\
\text { if there is } \\
\text { channel sharing } \\
\text { on TDMA: } \\
\text { scheme } \text { iii }\end{array}$ & $\begin{array}{l}\cong \text { scheme iii, } \\
\text { preferably TDMA }\end{array}$ \\
\hline
\end{tabular}

Figure 6 shows the results obtained with the optimal policy for the blocking optimization function, and the values obtained with the fixed policies. When $\lambda_{v}$ reaches 0.047 , the offered load for voice is $5.64 \mathrm{Erl}$, almost two times the offered load for data ( $3 \mathrm{Erl})$. Given the great impact on data calls, the optimal policy blocks voice calls, and this is more intense for higher values of $\lambda_{v}$. For $\lambda_{v}=0.047$ only 3 of 80 states where voice calls were sent to TDMA according to scheme ii, decide to block in the optimal policy. At this point, $B P_{v}=0.2 \%$ and $B P_{d}=1 \%$. When $\lambda_{v}$ grows to $0.065,26$ of those 80 states block voice calls, that is eight times more states than before. As expected, $B P_{d}$ only grows to $2.5 \%$, but the impact on $B P_{v}$ is bigger, since it grows almost ten times, to $2.056 \%$.

Dependance on channel sharing for $a_{s}^{d}$ is mantained for all $\lambda_{v}$, but once sharing is mandatory, some changes occur. As $\lambda_{v}$ grows, the number of states that decide to send data calls to TDMA under this circumstances, grows from 180 of 400 when $\lambda_{v}=0.005$ to 225 of 400 when $\lambda_{v}=0.065$. This is done in order to reduce $B P_{v}$, since WCDMA capacity is saved for voice calls. It is also worth noting that when TDMA is almost at full capacity $(>95 \%)$, data calls are blocked. This effect can be seen for the full range of $\lambda_{v}$ values that appear on Fig. 6 .

Figure 7 shows the throughput when it is used as the optimization function for the optimal policy and the three fixed policies as $\lambda_{v}$ grows. As $\lambda_{v}$ grows, it becomes mandatory to save resources for data calls since they have a higher throughput. For this reason, voice calls may be blocked even when there is room on TDMA. For $\lambda_{v}=0.005,14$ of 80 states where occupancy

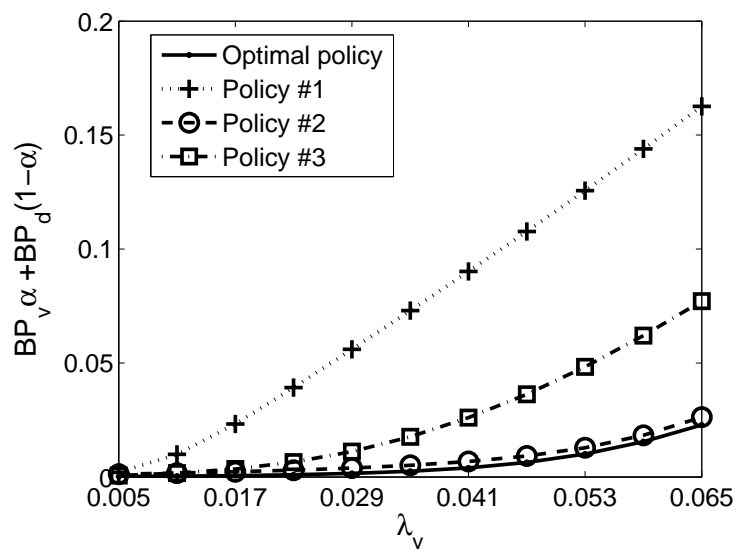

Figure 6: Blocking function for various $\lambda_{v}$.

on WCDMA is full but there is still room on TDMA, block voice calls. When $\lambda_{v}$ grows to $0.065,36$ of the same 80 states block voice calls. This affects $B P_{v}$, and when $\lambda_{v}>0.041$, the optimal policy has a higher $B P_{v}$ than Policy \#2.

Dependance on channel sharing for $a_{s}^{d}$ is mantained for all $\lambda_{v}$, but when sharing is mandatory, optimal policies vary. As $\lambda_{v}$ grows, the occupation of WCDMA does too, so more data calls must be sent to TDMA in order to increase the total throughput. When $\lambda_{v}=0.005$, 144 of the 400 states that use TDMA channel sharing send data calls to TDMA. For $\lambda_{v}=0.065,212$ of the same 400 states send calls to TDMA.

Therefore, for both optimization criteria, voice calls are sent to WCDMA, with more blocking states as $\lambda_{v}$ grows, and data calls are sent to TDMA while no sharing is needed. When sharing is mandatory, data calls may be sent to WCDMA or TDMA with more states using TDMA as $\lambda_{v}$ grows.

\subsection{Data users arrival rate variation.}

Although not identical, the main characteristics of the optimal policies for both optimization function as $\lambda_{d}$ grows can be summarized as: 


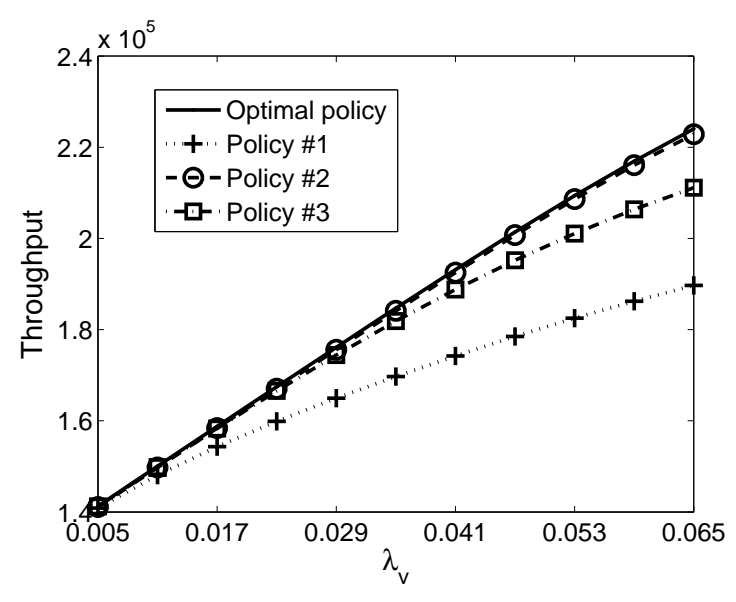

Figure 7: Throughput for various $\lambda_{v}$.

\begin{tabular}{|c|c|c|}
\hline Service & Lower $\lambda_{d}$ & Higher $\lambda_{d}$ \\
\hline Voice & scheme ii & scheme ii \\
\hline \multirow[t]{2}{*}{ Data } & $\begin{array}{l}\text { If there is no } \\
\text { channel sharing } \\
\text { on TDMA: } \\
\text { scheme } i\end{array}$ & $\begin{array}{l}\text { If there is no } \\
\text { channel sharing } \\
\text { on TDMA: } \\
\text { scheme } i\end{array}$ \\
\hline & $\begin{array}{l}\text { if there is } \\
\text { channel sharing } \\
\text { on TDMA: } \\
\cong \text { scheme iii }\end{array}$ & $\begin{array}{l}\text { if there is } \\
\text { channel sharing } \\
\text { on TDMA: } \\
\cong \text { scheme iii, } \\
\text { preferably WCDMA }\end{array}$ \\
\hline
\end{tabular}

Figure 8 compares the blocking function values for the optimal policy with different values of $\lambda_{d}$ with those obtained with the three fixed policies. In this scenario, voice calls follow scheme ii for every $\lambda_{d}$, showing that the load of data calls has no impact on $a_{s}^{v}$. On the other hand, the big influence of TDMA occupancy over $a_{s}^{d}$, suggest the opposite. In fact, when $\lambda_{d}=0.2$, data calls arrive with a higher rate than voice calls, so in order to mantain a low objective function value, it is necessary to send more data calls to WCDMA so TDMA's capacity is not exhausted too fast. This is the opposite case of the one we saw in the last section where as $\lambda_{v}$ grew, more data calls were sent to TDMA. The second effect is that as $\lambda_{d}$ grows, it is necessary to block calls in order to minimize the optimizing function. For the values of Fig. 8, the system has 1000 states. Only 70 of the total do not allow data calls, thus these are data blocking states. For the optimal policy, while $\lambda_{d} \leq 0.095$, data calls are only blocked on those 70 states. Once this value is surpased, the optimal policy may block data calls even when there is capacity left. When $\lambda_{d}=0.11,4$ of the 930 states with capacity left decide to block data

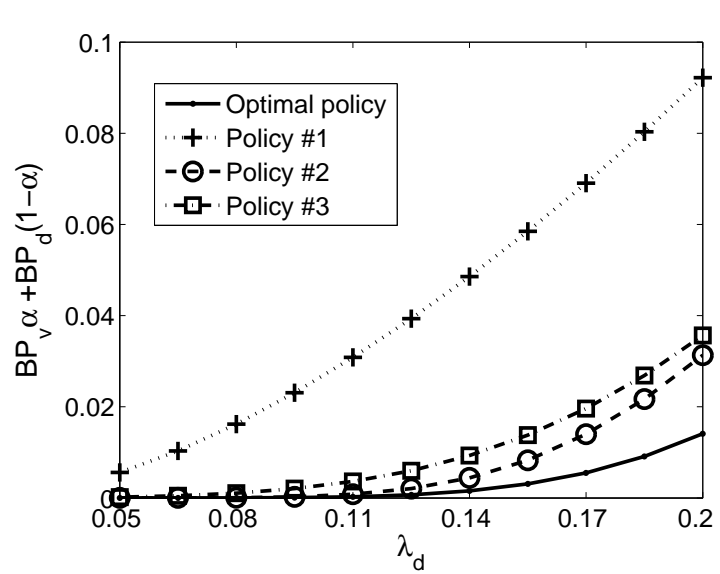

Figure 8: Blocking function for various $\lambda_{d}$.

calls, and this number grows to 30 of those 930 states when $\lambda_{d}=0.2$, more than seven times the initial number of blocking states.

Figure 9 shows the throughput when it is used as the optimization function for various $\lambda_{d}$. Since data calls have a higher throughput, the optimal policy may penalize some voice calls to maximize the objective function. When $\lambda_{d}=0.2, B P_{v}$ is higher in the optimal policy than in Policy \#2, reaching $2 \%$. In fact, if we let $\lambda_{d}=0.275, B P_{v}$ for the optimal policy would be higher than any of the fixed policies. In order to maximize the throughput, the opposite happens for $B P_{d}$, which is always the lowest in the optimal policy. As usual, voice calls are treated according to scheme ii, but as $\lambda_{d}$ grows higher than 0.05 , instead of sending calls to TDMA when WCDMA is full, they may be blocked. The number of states doing this grows slowly, but when $\lambda_{d}=0.3$ no voice calls are ever sent to TDMA, saving the space for data calls.

TDMA occupancy influence on $a_{s}^{d}$ is increased by $\lambda_{d}$. When sharing becomes mandatory, as $\lambda_{d}$ grows there seems to be a preference for sending calls to WCDMA using the capacity saved by voice calls. As a consequence of the importance of data calls for throughput, for the values of $\lambda_{d}$ in Fig.9, the optimal policy never blocks data calls unless there is no other chance. That is, as $\lambda_{d}$ grows voice calls are always sent to WCDMA, but some blocking may appear when we optimize the throughput. On the other hand $\lambda_{d}$ does not affect decisions for voice calls when the blocking function is optimized. Data calls are sent to TDMA while no sharing is needed, but when sharing is mandatory, calls may be sent to TDMA or WCDMA, but as $\lambda_{d}$ grows, a clear tendency towards WCDMA is seen for both optimiza- 


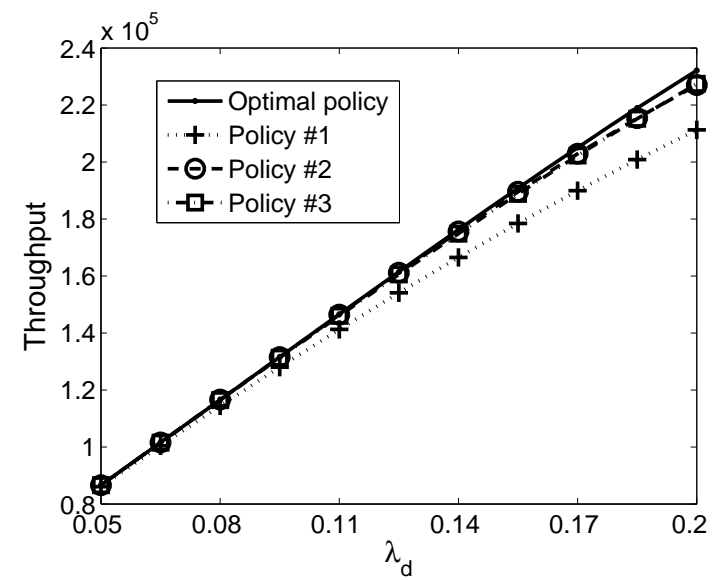

Figure 9: Throughput for various $\lambda_{d}$.

tion criteria.

\subsection{Voice users service rate variation}

Figure 10(a) shows the blocking function value while mantaining a constant offered traffic of $3 \mathrm{Erl}$ for both services (as the static scenario), and changing $\mu_{v}$ from 0.0833 to 1.833 . It is easy to realize how changes on $\mu_{v}$ have little or no impact in the blocking function for different policies. This behaviour is a result of what happens with the blocking probabilities and the throughput, where the results are almost constant for the full range of $\mu_{v}$. The optimal policies can be summarized as:

\begin{tabular}{|l|l|l|}
\hline Service & Lower $\mu_{v}$ & Higher $\mu_{v}$ \\
\hline Voice & scheme ii & $\begin{array}{l}\text { scheme } \text { ii with } \\
\text { blocking }\end{array}$ \\
\hline Data & $\begin{array}{l}\text { If there is no } \\
\text { channel sharing } \\
\text { on TDMA: } \\
\text { scheme } i\end{array}$ & $\begin{array}{l}\text { If there is no } \\
\text { channel sharing } \\
\text { on TDMA: } \\
\text { scheme } i\end{array}$ \\
\cline { 2 - 3 } & $\begin{array}{l}\text { if there is } \\
\text { channel sharing } \\
\text { on TDMA: } \\
\cong \text { scheme iii }\end{array}$ & $\begin{array}{l}\text { if there is } \\
\text { channel sharing } \\
\text { on TDMA: } \\
\cong \text { scheme iii. }\end{array}$ \\
\hline
\end{tabular}

As $\mu_{v}$ grows, some voice calls are blocked. However, it only differs on $0.2 \%$ of the states when $\mu_{v}$ changes from 0.258 to 1.833 . Also, $\mu_{v}$ has little influence over $a_{s}^{d}$. As $\mu_{v}$ grows, a small percentage of states $(<5 \%)$ where calls used to be sent to WCDMA are sent to TDMA. In Fig.10(b) it is shown the throughput as the optimizing function for a constant load of $3 \mathrm{Erl}$ in both

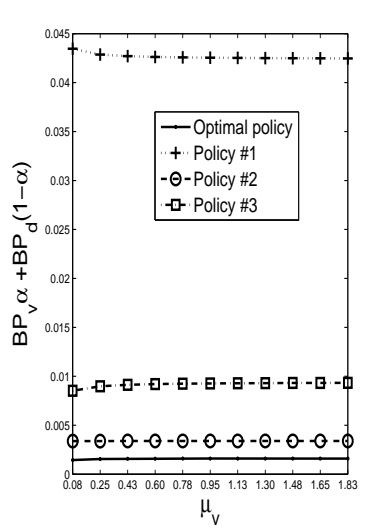

(a) Blocking Optimization.

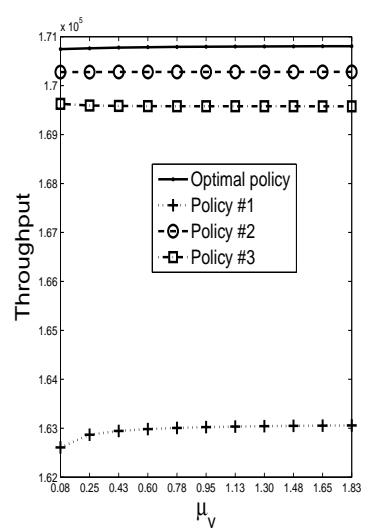

(b) Throughput Optimization.
Figure 10: Optimization for various $\mu_{v}$.

services, while $\mu_{v}$ changes. When throughput is optimized, $B P_{v}$ can be higher in the optimal policy than in other policies. The main characteristics of the optimal policies can be summarized in the same way as in the blocking function optimization shown before in this section.

\subsection{Data service rate variation}

Fig.11(a) shows the blocking function value for a constant load of $3 \mathrm{Erl}$ on both services while $\mu_{d}$ changes. To change this parameter, we kept $B R_{x, d}$ in $44.8 \mathrm{kbps}$, and changed the mean data length $\sigma$. The main characteristics of the optimal policies can be summarized as:

\begin{tabular}{|l|l|l|}
\hline Service & Lower $\mu_{d}$ & Higher $\mu_{d}$ \\
\hline Voice & scheme ii & $\begin{array}{l}\text { scheme ii with } \\
\text { blocking }\end{array}$ \\
\hline Data & $\begin{array}{l}\text { If there is no } \\
\text { channel sharing } \\
\text { on TDMA: } \\
\text { scheme } i\end{array}$ & $\begin{array}{l}\text { If there is no } \\
\text { channel sharing } \\
\text { on TDMA: } \\
\text { scheme } i\end{array}$ \\
\cline { 2 - 3 } & $\begin{array}{l}\text { if there is } \\
\text { channel sharing } \\
\text { if there is } \\
\text { channel sharing } \\
\text { on TDA: } \\
\text { on TDMA: } \\
\text { scheme iii }\end{array}$ & $\cong$ scheme iii. \\
\hline
\end{tabular}

In Fig.11(b) appears the throughput (used as the optimization function) for the optimal and the fixed policies when $\mu_{d}$ changes and the load for each service is kept constant $(3 \mathrm{Erl})$. The optimal policies are very similar to those obtained with the blocking function. For the 


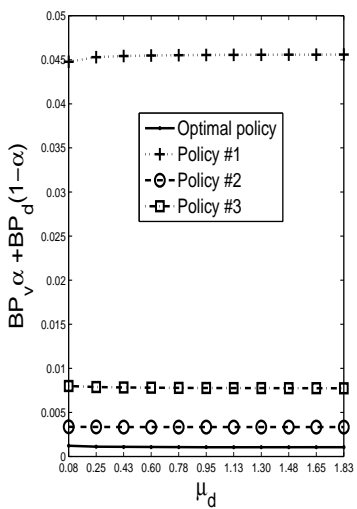

(a) Blocking Optimization.

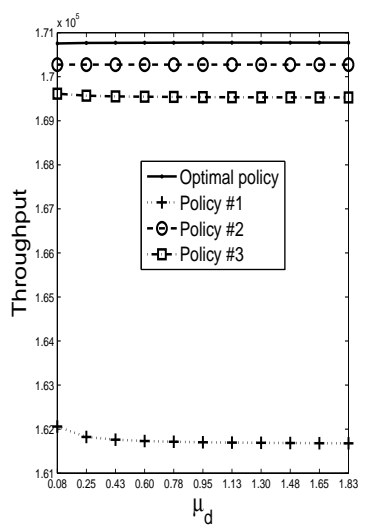

(b) Throughput Optimization.

Figure 11: Optimization for various $\mu_{d}$.

lowest values of $\mu_{d}$ in Fig.11(b) most of the states where the capacity of WCDMA is full, decide to block calls even when there is space left on TDMA. As $\mu_{d}$ grows, these states decide to send voice calls to TDMA. This blocking is done in order to protect data calls, whose contribution to throughput is higher.

\subsection{Heuristic policy}

According to the analysis realized in previous sections, it is possible to define a heuristic policy based on those characteristics that were common for all the scenarios.

Therefore, the heuristic policy is summarized as:

\begin{tabular}{|c|l|}
\hline Service & action \\
\hline Voice & scheme ii \\
\hline Data & $\begin{array}{l}\bullet \text { if no channel sharing } \\
\text { on TDMA: scheme } i \\
\bullet \text { if channel sharing } \\
\text { on TDMA: scheme iii }\end{array}$ \\
\hline
\end{tabular}

The heuristic policy sends voice calls to WCDMA which is a very simple solution that is consistent with optimal solutions of Section 5.2 and for the lower values of $\lambda_{v}$ in Section 5.1. On the other hand, the optimal solution for data calls is more complex and composed by two stages as previous analysis showed. On the first stage, data calls are sent to TDMA (scheme 1) until the arrival of a new call would force channel sharing. On the second stage decisions depend on voice and data loads, as can be seen from Sections 5.1 and 5.2. When

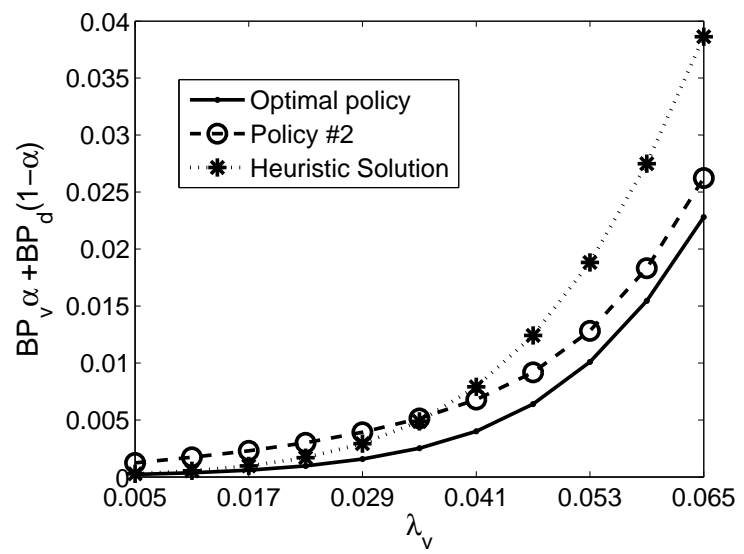

Figure 12: Blocking function for various $\lambda_{v}$.

$\lambda_{v}$ grows, data calls are sent to TDMA, making the optimal policy for data calls as scheme 1 . On the other hand, when $\lambda_{d}$ is the one that grows, more data calls are sent to WCDMA. Therefore the heuristic policy uses scheme 1 for the first stage, and scheme 3 for the second stage, that is, compares the occupancy on each technology and sends calls to the one with the lowest value.

Figure 12 compares the blocking function of the heuristic policy and Policy \#2 for the same values of Fig.5. It is evident that for the lowest values of $\lambda_{v}$, the heuristic policy is better than Policy \#2, but this changes when $\lambda_{v}>0.035$. This occurs because Policy \#2 always sends calls to TDMA, and this behaviour is similar to what is done by the optimal solution as $\lambda_{v}$ grows (Section 5.1). The same could be said for Fig.13, where the throughput is the optimization function. In this case the improvement of the heuristic solution over Policy \#2 is very small, but it is mantained while $\lambda_{v}<0.041$. When this value is reached, Policy \#2 is better than the heuristic solution because of its similarity to the optimal solution.

Figure 14 compares the heuristic solution with Policy \#2 and the optimal solution, when the blocking function is optimized and $\lambda_{d}$ changes. In this case, for the lowest values of $\lambda_{d}$ Policy \#2 is better than the heuristic, but once $\lambda_{d}>0.08$, the heuristic is better. This can be explained by the fact that the optimal solution sends more data calls to WCDMA as $\lambda_{d}$ grows, and while Policy \#2 always sends data calls to TDMA, the heuristic policy may send some calls to WCDMA according to occupation on each technology.This behaviour has the same effect over the throughput as can be seen on Fig.15, where it is compared the throughput for the heuristic solution and Policy \#2. For the lowest values 


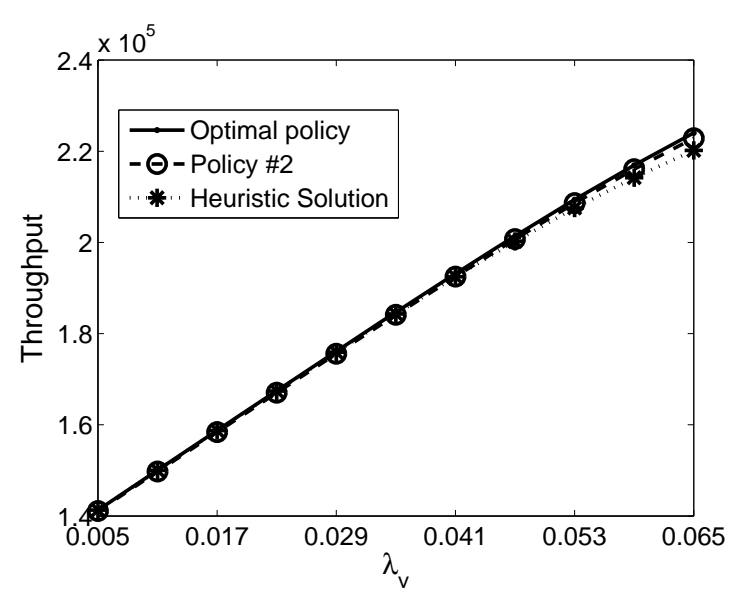

Figure 13: Throughput for various $\lambda_{v}$.

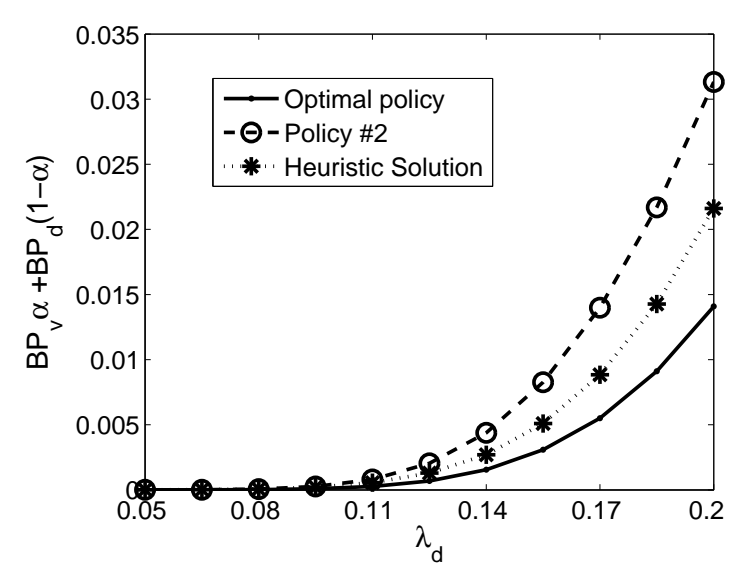

Figure 14: Blocking function for various $\lambda_{d}$.

of $\lambda_{d}$, Policy \#2 is better than the heuristic, but when $\lambda_{d}>0.65$ the heuristic solution has a higher throughput. Therefore the heuristic policy represents some advantages over the best fixed policy when data load is high, but its behaviour is not as good as that of Policy \#2 when voice load is high.

\section{Throughput optimization with QoS constraints}

In this section we compare the policies in terms of the maximum arrival they can support subject to QoS constraints. Until now, we considered throughput optimization independent of other factors, but to provide QoS, it is necessary to limit both $P B_{v}$ and $P B_{d}$. To do this, we defined the parameter $\beta$, which is the fraction of $\lambda_{v}$ over the total arrival rate $\lambda_{T}$, that is, $\lambda_{v}=\lambda_{T} \cdot \beta$ and $\lambda_{d}=\lambda_{T} \cdot(1-\beta)$. In Fig.16 it is shown the throughput as a function of $\beta$ for three policies: The optimal policy

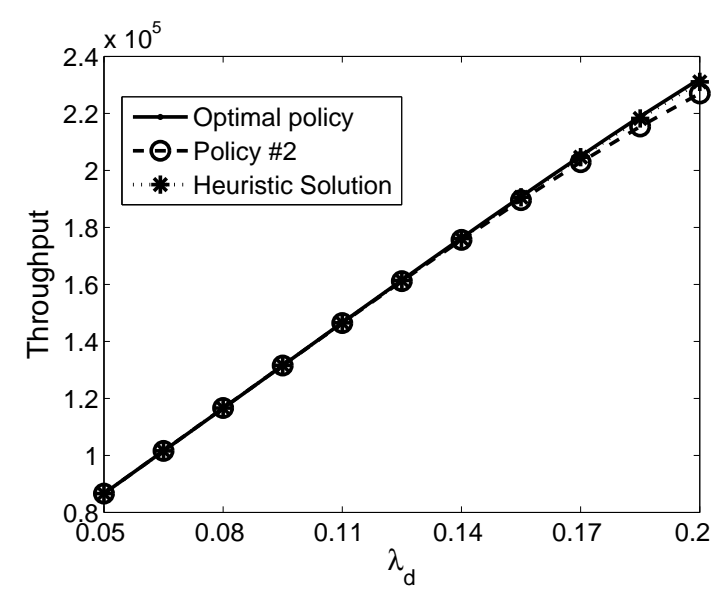

Figure 15: Throughput for various $\lambda_{d}$.

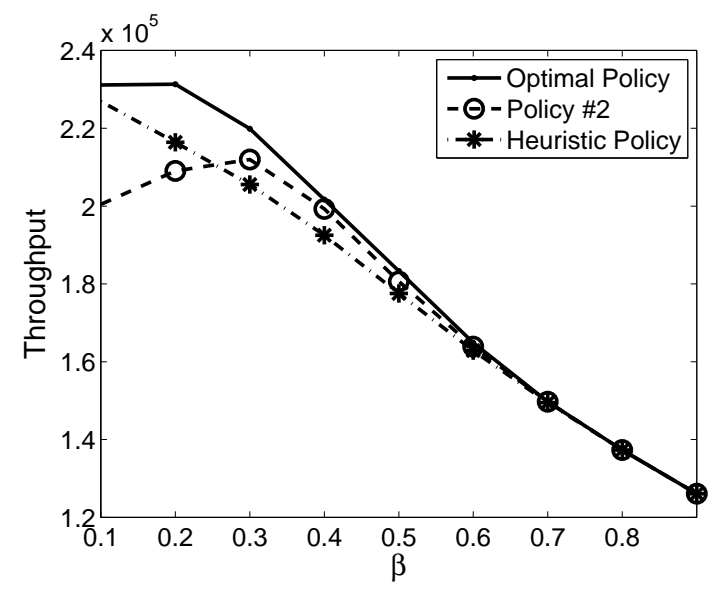

Figure 16: Throughput as a function of $\beta$. 


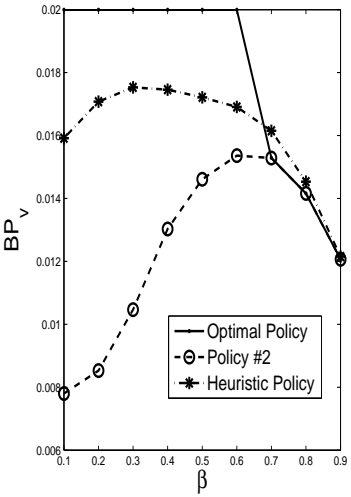

(a) VoiceBlocking Probability.

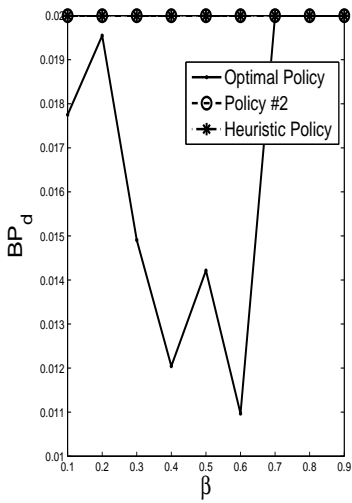

(b) DataBlocking Probability.
Figure 17: Blocking probabilities for various $\beta$.

when we optimize the throughput, Policy \#2, and the heuristic policy defined in the last section. It has to be considered that even when they share the same value of $\beta$, the arrival rates differ for each policy according to the restrictions imposed by the blocking probabilites (max $2 \%$ ). For example, when $\beta=0.2$, the total arrival rate $\lambda_{T}$ that the optimal policy can handle is 0.2158 , while it is of 0.2018 for the heuristic policy and 0.1945 for Policy \#2. Therefore, the optimal policy is able to receive a higher arrival rate for voice and data calls while mantaining $P B_{v}$ and $P B_{d}$ below $2 \%$. Figures 17(a) and 17(b) show the voice and data blocking probabilities for different values of $\beta$. It can be seen that the optimal policy is the only one able to change its active constraint between $P B_{v}$ and $P B_{d}$, taking advantage of the contribution of data calls on the throughput. This cannot be done by the other two policies, which penalize $P B_{d}=0.2$ for every $\beta$. As for the heuristic policy, it shows a higher maximum throughput for low values of $\beta$ than Policy \#2, and this behaviour is reversed as $\beta$ grows when voice calls are more important in order to maximize throughput, a behaviour consistent with the results of the previous section.

\section{Optimization with vertical handoff}

So far, we have analyzed a system where no vertical handoff is used (now referred to as NVH), i.e. a call is served in the same technology where it was accepted. However, vertical handoff is considered an important feature in common radio resource management that improves the use of resources. In Table 4 we introduce four types of vertical handoff based on the analysis done in

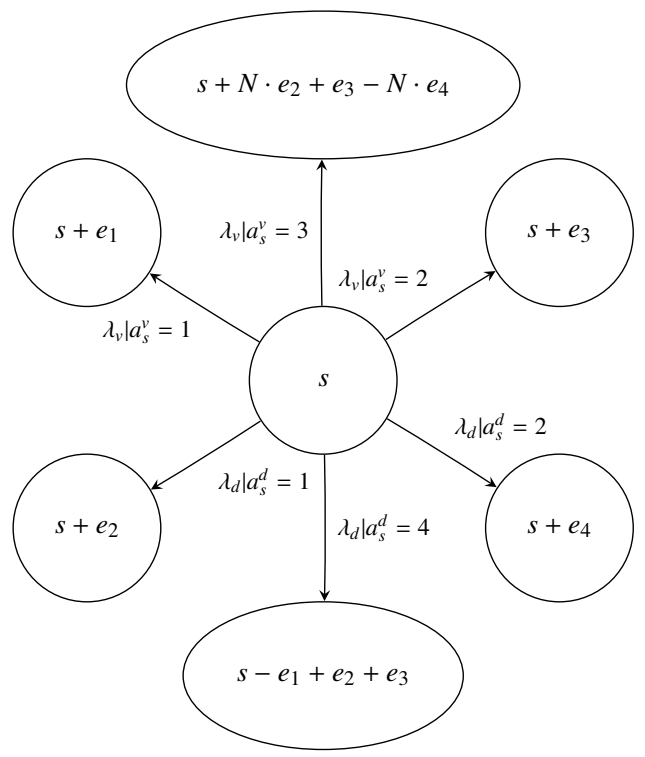

Figure 18: Transitions for voice and data arrivals using VH.

previous sections, where $N$ is the necessary number of data calls such that one voice call can access WCDMA. All types of vertical handoff are based on the results obtained in Sections 4 and 5, but the first two types are triggered by the arrival of a call, while the other two occur when a call is served. This distinction is important, since it affects the construction of the new Markov decission processes. The addition of types I and II of vertical handoff to NVH defines VH-A. The Markov model for this system has the same state space $S$ of NVH, since restrictions (1) and (2) are not affected by vertical handoff. The same applies for the Markov model of system VH-B, which uses all types of vertical handoff (I-IV). However, the set of actions $A$ and the transitions associated to them do change. For VH-A, a decision is made each time a call arrives as can be seen in Table 5 and in Figure 18, where actions 0,1 and 2 do not include vertical handoff, while actions 3 and 4 correspond to vertical handoff types I and II respectively. For action 3 , the value $N$ is the number of data calls that need to be moved so that a new voice call can access WCDMA according to (2). The same applies for system VH-B, which includes vertical handoff types III and IV. The reason why handoff types III and IV do not affect the set $A$ is that decisions are made only when a call arrives. In our model, handoffs triggered by service completion are done every time their specific conditions are fulfilled, so there are no decisions involved.

With this in mind, and using the same cost functions of 


\begin{tabular}{|c|c|c|c|c|c|c|c|}
\hline Type & Triggering Event & Conditions & $\begin{array}{c}\text { Number } / \\
\text { Class of calls }\end{array}$ & From $\Rightarrow$ To & NVH & VH-A & $V H-B$ \\
\hline I & voice arrival & $\begin{array}{c}\text { Full occupation } \\
\text { on WCDMA }\end{array}$ & $N$ data calls & WCDMA $\Rightarrow$ TDMA & - & $\checkmark$ & $\checkmark$ \\
\hline II & data arrival & $\begin{array}{c}\text { Channel sharing } \\
\text { on TDMA }\end{array}$ & One voice call & TDMA $\Rightarrow$ WCDMA & - & $\checkmark$ & $\checkmark$ \\
\hline III & $\begin{array}{c}\text { voice or data } \\
\text { departure from } \\
\text { WCDMA }\end{array}$ & $\begin{array}{c}\text { Channel sharing } \\
\text { on TDMA }\end{array}$ & One voice call & TDMA $\Rightarrow$ WCDMA & - & - & $\checkmark$ \\
\hline IV & $\begin{array}{c}\text { voice or data } \\
\text { departure from } \\
\text { TDMA }\end{array}$ & $\begin{array}{c}\text { VH does not } \\
\text { produce channel } \\
\text { sharing on TDMA }\end{array}$ & One data call & WCDMA $\Rightarrow$ TDMA & - & - & $\checkmark$ \\
\hline
\end{tabular}

Table 4: Vertical Handoff Types

(4) and (5), two new MDPs are constructed. These new MDPs will show the improvement when vertical handoff is included, where the MDP based on VH-A uses handoff when calls arrive and the MDP based on VH-B uses handoff for both arrivals and departures.

\subsection{Optimal policy using vertical handoff}

In the last section were introduced two new systems that included vertical handoff: VH-A and VH-B. The difference between them is that VH-B includes vertical handoff for users departures. In this section we use two new MDPs that are based on VH-A (MDP VH-A) and VH-B (MDP VH-B), and compare results with those obtained with the MDP for the NVH system (MDP NVH). It is our interest to study the impact that vertical handoff has over the optimal solutions and performance. Using the scenario specified in Table 3 for MDP VH-A and MDP VH-B, it is possible to extract the main characteristics of the optimal solutions as different parameters vary. Results for optimization of the blocking function defined in (4) while $\lambda_{v}$ varies from 0.005 to 0.095 are summarized in Table 6 . In general, the behaviour for voice and data calls are very similar in both MDPs. Nevertheless, some minor differences change the structure of the optimal solutions, and that has an important impact on the results obtained. Optimal solutions for MDP VH-A show a lower percentage of unused states than those solutions obtained using MDP VH-B. This is because Vertical Handoff types III and IV organize calls in such a way that a larger number of states from set $S$ are left unused. When $\lambda_{v}=0.005$ the percentage of unused states is three times higher for the solution obtained with MDP VH-B
Table 5: set of actions A for MDPs with vertical handoff

\begin{tabular}{|c|l|}
\hline value & action \\
\hline 0 & Block call \\
1 & Send call to TDMA \\
2 & Send call to WCDMA \\
3 & VH for N data calls from WCDMA \\
& to TDMA and the voice call is \\
& sent to WCDMA. \\
4 & VH for 1 voice call from TDMA \\
& to WCDMA and the data call is \\
& sent to TDMA. \\
\hline
\end{tabular}

than for the one obtained with MDP VH-A, and while this difference shortens when $\lambda_{v}=0.095$, the percentage of unused states grows. This growth shows that an organized use of resources is necessary in order to obtain the optimal solution in both MDPs. Also, it may occur some voice blocking when there are resources available, but this behaviour is expected only for the highest values of $\lambda_{v}$, when its influence is higher on the optimization function.

Similar results are obtained for optimization of the blocking function defined in (4) when $\lambda_{d}$ varies from 0.05 to 0.225 . Voice and data calls use Vertical Handoff types I and II respectively, but the percentage of unused states follows a different pattern. As $\lambda_{d}$ grows, the percentage of unused states for the optimal solutions obtained with MDP VH-A remains constant, and it decreases for the solutions of MDP VH-B. This decrease 
shows that Vertical Handoff types III and IV allow more flexibility on how resources are managed, because a higher data arrival rate demands that more data calls share channels in order to reduce the blocking probability, something that cannot be done as successfully by only using Vertical Handoff types I and II. Also, for the highest values of $\lambda_{d}$, some states decide to block data calls even when there is capacity left, in order to save space for voice calls. However, the percentage of states doing this is below 3\% for both MDPs. A summary of these results is shown in Table 7.

Using the scenario specified in Table 3 and the throughput optimization function defined in (5) while $\lambda_{v}$ varies from 0.005 to 0.095 , the optimal policies for MDP VH-A and MDP VH-B are summarized in Table 8.Again, voice and data calls use vertical handoff types I and II respectively in both MDPs. For the lowest values of $\lambda_{v}$, both MDPs decide to block voice calls $(<1 \%)$ in order to enhance throughput, since data calls contribute more to the total throughput. However, this situation changes as $\lambda_{v}$ grows when voice calls are accepted as long as it is possible. On the other hand, the percentage of unused states differs for each MDP. For MDP VH-A, the percentage of unused states grows with $\lambda_{v}$ from $16 \%$ to $41.7 \%$. On the other hand, for MDP VH-B, the percentage of unused states diminishes from $61.2 \%$ to $49.5 \%$ as $\lambda_{v}$ grows.

The optimal policies for MDP VH-A and MDP VH-B as $\lambda_{d}$ varies from 0.05 to 0.225 are summarized in Table 9.Voice and data calls use vertical handoff types I and II respectively in both MDPs. For the highest values of $\lambda_{d}$ MDPs decide to block some voice calls, even though in a very small percentage $(<1 \%)$. This is done in order to accept more data calls since they contribute more to the total throughput. For both MDPs the percentage of unused states grows with $\lambda_{d}$, and it is always higher for MDP VH-B.

\subsection{Result analysis for vertical handoff MDPs}

Considering the optimal policies obtained in the previous section for MDP VH-A and MDP VH-B, we propose two new heuristic policies that make use of their main characteristics. Heuristic VH-A makes use of vertical handoff types I and II, as it was seen for the optimal policies found using MDP VH-A. On the other hand, Heuristic VH-B also includes vertical handoff types III and IV, as it was done by the optimal policies obtained
Table 11: New study Scenario.

\begin{tabular}{|c|c|}
\hline WCDMA & TDMA \\
\hline$W=3.84 \mathrm{Mcps}$ & $C=8$ \\
$\left(E_{b} / N_{0}\right)_{v}=6.5 \mathrm{~dB}$ & $n_{c}=3$ \\
$\left(E_{b} / N_{0}\right)_{d}=5 \mathrm{~dB}$ & $B R_{t, v}=12.2 \mathrm{kbps}$ \\
$B R_{w, v}=12.2 \mathrm{kbps}$ & $B R_{t, d}=44.8 \mathrm{kbps}$ \\
$B R_{w, d}=44.8 \mathrm{kbps}$ & \\
$\eta_{u l}=1$ & \\
\hline \hline Clients \\
\hline$\mu_{v}=0.0083$ \\
$\sigma=1 \mathrm{Mb}$ \\
\hline
\end{tabular}

using MDP VH-B. In this section we compare the results obtained by the two new heuristic policies with the optimal solutions of the MDPs and the three fixed policies that were explained earlier for the system described in Table 11. For this system, the maximum capacity for voice and data calls in WCDMA are 71 and 28 respectively, so the capacity has grown over 6 times over the system described in Table 3 . However, the computational cost does not grow linearly. Using a 2 Quad CPU @2.4GHz, 3.24 GB RAM, it took around 1 minute to find the optimal solution of a single load point for the smaller system, while it took around 6 hours in this system. Therefore, the calculation time of the optimal solutions makes them unfeasible for on-line use. It should be noted that this is not an issue for the heuristic schemes that can be used as a JCAC algorithm.

In Fig. 19 it is shown the blocking function for the three MDPs, the two heuristics and the three fixed policies when $\lambda_{v}$ varies from 0.09996 to 0.4998 and $\lambda_{d}=0.448$. The blocking function value when $\lambda_{v}=0.4998$ is reduced to less of the half of the value of Policy \#2 when the heuristic policies are used, and this improvement is more visible when comparing to the other fixed policies. Also, it should be noted that the heuristic policies may even improve over the optimal solution of MDP NVH as $\lambda_{v}$ grows. The improvement of the blocking function has an effect in the aggregated throughput, which raises to $1.156 \mathrm{Mbps}$ for Heuristic VH-A while it only reaches $1.116 \mathrm{Mbps}$ for Policy \#2 when $\lambda_{v}=0.4998$. Therefore under these conditions, using an heuristic policy represents an improvement of $40 \mathrm{kbps}$, close to one data channel, or 3 voice channels, with blocking probabilities of $2.2 \%$ and $1.8 \%$ for data and voice, while Policy \#2 raises them to $6.5 \%$ and $4.6 \%$, respectively. 


\begin{tabular}{|c|c|c|c|}
\hline & & MDP VH-A & MDP VH-B \\
\hline \multirow[t]{2}{*}{ Voice calls } & Lowest $\lambda_{v}$ & $\begin{array}{l}- \text { Sent to WCDMA or VH } \\
\text { type I is used. } \\
-10.4 \% \text { of states are not used. }\end{array}$ & $\begin{array}{l}\text { - Sent to WCDMA or VH } \\
\text { type I is used. } \\
\text { - } 32.1 \% \text { of states are not used. }\end{array}$ \\
\hline & Highest $\lambda_{v}$ & $\begin{array}{l}\text { - Sent to WCDMA or VH } \\
\text { type I is used. } \\
\text { - } 41.7 \% \text { of states are not used. } \\
\text { - Blocked in } 1.00 \% \text { of usable } \\
\text { states. }\end{array}$ & $\begin{array}{l}\text { - Sent to WCDMA or VH } \\
\text { type I is used. } \\
\text { - } 50.4 \% \text { of states are not used. } \\
\text { - Blocked in } 1.2 \% \text { of usable } \\
\text { states. }\end{array}$ \\
\hline \multirow[t]{2}{*}{ Data calls } & Lowest $\lambda_{v}$ & $\begin{array}{l}- \text { Sent to TDMA while no } \\
\text { sharing is needed. If so, VH } \\
\text { type II is used or sent to } \\
\text { WCDMA. } \\
-10.4 \% \text { of states are not used. }\end{array}$ & $\begin{array}{l}\text { - Sent to TDMA while no } \\
\text { sharing is needed. If so, VH } \\
\text { type II is used or sent to } \\
\text { WCDMA. } \\
\text { - } 32.1 \% \text { of states are not used. }\end{array}$ \\
\hline & Highest $\lambda_{v}$ & $\begin{array}{l}- \text { Sent to TDMA while no } \\
\text { sharing is needed. If so, VH } \\
\text { type II is used or sent to } \\
\text { WCDMA. } \\
-41.7 \% \text { of states are not used. }\end{array}$ & $\begin{array}{l}\text { - Sent to TDMA while no } \\
\text { sharing is needed. If so, VH } \\
\text { type II is used or sent to } \\
\text { WCDMA. } \\
-50.4 \% \text { of states are not used. }\end{array}$ \\
\hline
\end{tabular}

Table 6: Main characteristics of the optimal solutions for the blocking function for various $\lambda_{v}$.

\begin{tabular}{|c|c|c|c|}
\hline & & MDP VH-A & MDP VH-B \\
\hline \multirow[t]{2}{*}{ Voice calls } & Lowest $\lambda_{d}$ & $\begin{array}{l}\text { - Sent to WCDMA or VH } \\
\text { type I is used. } \\
-41.6 \% \text { of states are not used. }\end{array}$ & $\begin{array}{l}\text { - Sent to WCDMA or VH } \\
\text { type I is used. } \\
-49.4 \% \text { of states are not used. }\end{array}$ \\
\hline & Highest $\lambda_{d}$ & $\begin{array}{l}\text { - Sent to WCDMA or VH } \\
\text { type I is used. } \\
\text { - } 41.6 \% \text { of states are not used. }\end{array}$ & $\begin{array}{l}\text { - Sent to WCDMA or VH } \\
\text { type I is used. } \\
\text { - } 39.6 \% \text { of states are not used. }\end{array}$ \\
\hline \multirow[t]{2}{*}{ Data calls } & Lowest $\lambda_{d}$ & $\begin{array}{l}\text { - Sent to TDMA while no } \\
\text { sharing is needed. If so, VH } \\
\text { type II is used or sent to } \\
\text { WCDMA. } \\
\text { - } 41.6 \% \text { of states are not used. }\end{array}$ & $\begin{array}{l}- \text { Sent to TDMA while no } \\
\text { sharing is needed. If so, VH } \\
\text { type II is used or sent to } \\
\text { WCDMA. } \\
\text { - } 49.4 \% \text { of states are not used. }\end{array}$ \\
\hline & Highest $\lambda_{d}$ & $\begin{array}{l}\text { - Sent to TDMA while no } \\
\text { sharing is needed. If so, VH } \\
\text { type II is used or sent to } \\
\text { WCDMA. } \\
\text { - } 41.6 \% \text { of states are not used. } \\
\text { - Blocked in } 2.56 \% \text { of usable } \\
\text { states. }\end{array}$ & $\begin{array}{l}\text { - Sent to TDMA while no } \\
\text { sharing is needed. If so, VH } \\
\text { type II is used or sent to } \\
\text { WCDMA. } \\
\text { - } 39.6 \% \text { of states are not used. } \\
\text { - Blocked in } 2.98 \% \text { of usable } \\
\text { states. }\end{array}$ \\
\hline
\end{tabular}

Table 7: Main characteristics of the optimal solutions for the blocking function for various $\lambda_{d}$.

In Fig. 20 is shown the blocking function for the MDPs, the heuristics and the fixed policies when $\lambda_{d}$ varies from 0.3584 to 1.792 and $\lambda_{v}$ is 0.0833 . The blocking function values are lower for the heuristic functions when compared to the fixed policies, but they are higher than those obtained by MDP NVH 


\begin{tabular}{|c|c|c|c|}
\hline & & MDP VH-A & MDP VH-B \\
\hline \multirow[t]{2}{*}{ Voice calls } & Lowest $\lambda_{v}$ & $\begin{array}{l}\text { - Sent to WCDMA or VH } \\
\text { type I is used. } \\
\text { - } 16 \% \text { of states are not used. } \\
\text { - Blocked in } 0.71 \% \text { of usable } \\
\text { states. }\end{array}$ & $\begin{array}{l}\text { - Sent to WCDMA or VH } \\
\text { type I is used. } \\
\text { - } 61.2 \% \text { of states are not used. } \\
\text { - Blocked in } 0.25 \% \text { of usable } \\
\text { states. }\end{array}$ \\
\hline & Highest $\lambda_{v}$ & $\begin{array}{l}- \text { Sent to WCDMA or VH } \\
\text { type I is used. } \\
-41.7 \% \text { of states are not used. }\end{array}$ & $\begin{array}{l}\text { - Sent to WCDMA or VH } \\
\text { type I is used. } \\
\text { - } 49.5 \% \text { of states are not used. }\end{array}$ \\
\hline \multirow[t]{2}{*}{ Data calls } & Lowest $\lambda_{v}$ & $\begin{array}{l}- \text { Sent to TDMA while no } \\
\text { sharing is needed. If so, VH } \\
\text { type II is used or sent to } \\
\text { WCDMA. } \\
\text { - } 16 \% \text { of states are not used. }\end{array}$ & $\begin{array}{l}- \text { Sent to TDMA while no } \\
\text { sharing is needed. If so, VH } \\
\text { type II is used or sent to } \\
\text { WCDMA. } \\
-61.2 \% \text { of states are not used. }\end{array}$ \\
\hline & Highest $\lambda_{v}$ & $\begin{array}{l}\bullet \text { Sent to TDMA while no } \\
\text { sharing is needed. If so, VH } \\
\text { type II is used or sent to } \\
\text { WCDMA. } \\
\bullet 41.7 \% \text { of states are not used. }\end{array}$ & $\begin{array}{l}\text { - Sent to TDMA while no } \\
\text { sharing is needed. If so, VH } \\
\text { type II is used or sent to } \\
\text { WCDMA. } \\
-49.5 \% \text { of states are not used. }\end{array}$ \\
\hline
\end{tabular}

Table 8: Main characteristics of the optimal solutions for throughput for various $\lambda_{v}$.

\begin{tabular}{|c|c|c|c|}
\hline & & MDP VH-A & MDP VH-B \\
\hline \multirow[t]{2}{*}{ Voice calls } & Lowest $\lambda_{d}$ & $\begin{array}{l}\text { - Sent to WCDMA or VH } \\
\text { type I is used. } \\
-41.6 \% \text { of states are not used. }\end{array}$ & $\begin{array}{l}\text { - Sent to WCDMA or VH } \\
\text { type I is used. } \\
-45.4 \% \text { of states are not used. }\end{array}$ \\
\hline & Highest $\lambda_{d}$ & $\begin{array}{l}\text { - Sent to WCDMA or VH } \\
\text { type I is used. } \\
\text { - } 43 \% \text { of states are not used. } \\
\text { - Blocked in } 0.7 \% \text { of usable } \\
\text { states. }\end{array}$ & $\begin{array}{l}\text { - Sent to WCDMA or VH } \\
\text { type I is used. } \\
\text { - } 47.2 \% \text { of states are not used. } \\
\text { - Blocked in } 0.56 \% \text { of usable } \\
\text { states. }\end{array}$ \\
\hline \multirow[t]{2}{*}{ Data calls } & Lowest $\lambda_{d}$ & $\begin{array}{l}- \text { Sent to TDMA while no } \\
\text { sharing is needed. If so, VH } \\
\text { type II is used or sent to } \\
\text { WCDMA. } \\
\text { - } 41.6 \% \text { of states are not used. }\end{array}$ & $\begin{array}{l}- \text { Sent to TDMA while no } \\
\text { sharing is needed. If so, VH } \\
\text { type II is used or sent to } \\
\text { WCDMA. } \\
-45.4 \% \text { of states are not used. }\end{array}$ \\
\hline & Highest $\lambda_{d}$ & $\begin{array}{l}\text { - Sent to TDMA while no } \\
\text { sharing is needed. If so, VH } \\
\text { type II is used or sent to } \\
\text { WCDMA. } \\
-43 \% \text { of states are not used. }\end{array}$ & $\begin{array}{l}- \text { Sent to TDMA while no } \\
\text { sharing is needed. If so, VH } \\
\text { type II is used or sent to } \\
\text { WCDMA. } \\
-47.2 \% \text { of states are not used. }\end{array}$ \\
\hline
\end{tabular}

Table 9: Main characteristics of the optimal solutions for throughput for various $\lambda_{d}$.

when $\lambda_{d}>1.0752$. However, when $\lambda_{d}=1.792$, the total throughput for heuristic VH-A is of $1.4896 \mathrm{Mbps}$ and of 1.466 Mbps for MDP NVH. That is, the heuristic solu- tions cannot diminish the value of the blocking function for the higher values of $\lambda_{d}$ as much as MDP NVH does, but they still raise the throughput, even though this is not 


\begin{tabular}{|c|c|c|}
\hline Heuristic & Event & Action \\
\hline \multirow[t]{2}{*}{ Heuristic VH-A } & Voice arrival & $\begin{array}{l}\text { - Send to WCDMA. } \\
\text { - If it is not possible, use VH type I. } \\
\text { - If it is not possible, send to TDMA. }\end{array}$ \\
\hline & Data arrival & $\begin{array}{l}\text { - Send to TDMA if no channel sharing is needed. } \\
\text { - If there is channel sharing, use VH type II. } \\
\text { - If it is not possible, send to WCDMA. } \\
\text { - If it is not possible, send to TDMA. }\end{array}$ \\
\hline \multirow[t]{4}{*}{ Heuristic VH-B } & Voice arrival & $\begin{array}{l}\text { - Send to WCDMA. } \\
\text { - If it is not possible, use VH type I. } \\
\text { - If it is not possible, send to TDMA. }\end{array}$ \\
\hline & Data arrival & $\begin{array}{l}\text { - Send to TDMA if no channel sharing is needed. } \\
\text { - If there is channel sharing, use VH type II. } \\
\text { - If it is not possible, send to WCDMA. } \\
\text { - If it is not possible, send to TDMA. }\end{array}$ \\
\hline & Voice departure & $\begin{array}{l}\text { - Use VH type III if departs from WCDMA } \\
\text { - Use VH type IV if departs from TDMA }\end{array}$ \\
\hline & Data departure & $\begin{array}{l}\text { - Use VH type III if departs from WCDMA } \\
\text { - Use VH type IV if departs from TDMA }\end{array}$ \\
\hline
\end{tabular}

Table 10: Definition for Heuristic policies with vertical handoff.

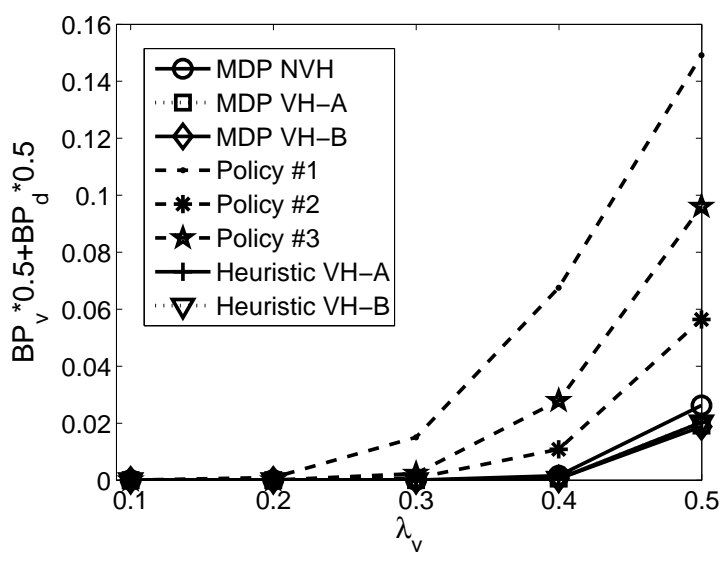

Figure 19: Blocking function for various $\lambda_{v}$.

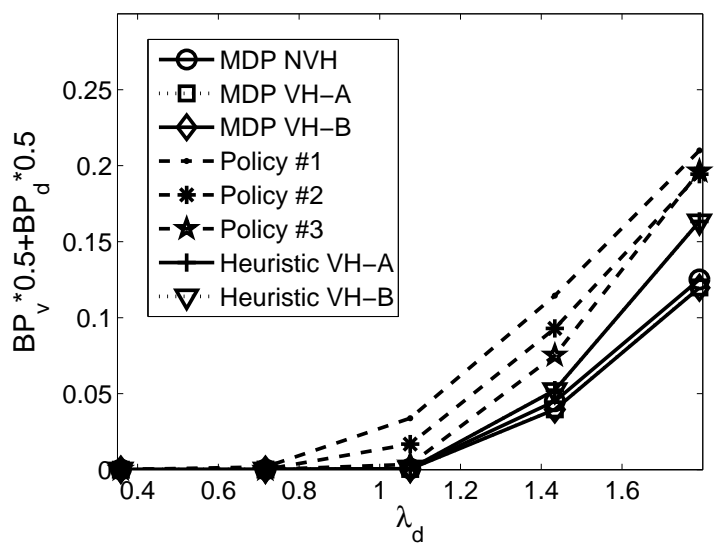

Figure 20: Blocking function for various $\lambda_{d}$.

the objective function. As in the case when $\lambda_{v}$ varies, the differences for both heuristics are not very significant.

The optimal throughput for the MDPs, as well as the throughput obtained by the heuristic solutions and the fixed policies as $\lambda_{v}$ grows from 0.09996 to 0.4998 keeping $\lambda_{d}=0.448$ is shown in Fig. 21. There is an improvement of throughput of the heuristic solutions not only over the fixed policies, but also over the optimal solutions of MDP NVH. Although the improve- 


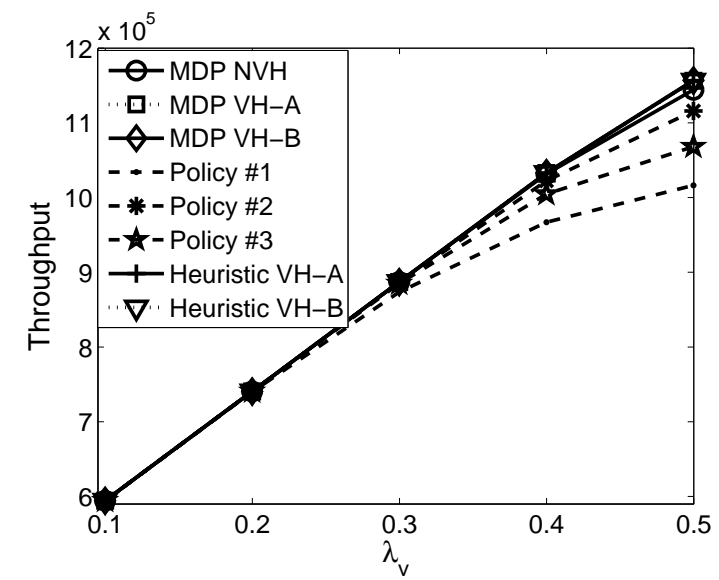

Figure 21: Throughput for various $\lambda_{v}$.

ment in throughput is small when $\lambda_{v}=0.4998,12 \mathrm{kbps}$ over MDP NVH and more when compared to the fixed policies, it is significant when we consider that the voice and data blocking probabilities of Heuristic VH-A are of $2.2 \%$ and $1.8 \%$ respectively, while these probabilities are of $1.5 \%$ and $3.8 \%$ for MDP NVH, and raise to a range of $6.5-19.5 \%$ and $4.6-10.5 \%$ for the fixed policies. That is, the heuristic solutions are able to improve throughput while mantaining low blocking probabilites (around 2\%), and the other policies are not.

In Fig. 22 is shown the throughput for the MDPs, the heuristics and the fixed policies when $\lambda_{d}$ varies from 0.3584 to 1.792 and $\lambda_{v}$ is 0.0833 . It is shown that there is not to much room for improvement of the throughput when $\lambda_{d}$ varies, since most solutions obtain similar values. The only real difference is seen when $\lambda_{d}=1.792$, when the MDPs raise around $6 \mathrm{kbps}$ over the other solutions, but since this is done blocking all the voice calls, this solutions cannot be considered useful. It is interesting to see that that the heuristics still achieve a higher throughput than all the fixed policies, while keeping lower voice and data blocking probabilities. However, when $\lambda_{d}=1.4336$, the voice and data blocking probabilities of the heuristic solutions are of $5 \%$ and $2.9 \%$ for data and voice, thus the load limit lies around these values. A closer look at the performance of the heuristic solutions shows that any degradation when compared to the optimal solutions behaviour is negligible, and this performance is consistent for both optimization criteria and several system's parameters.

\subsection{Model Validation}

In order to validate the solutions found by the Markov model, we have evaluated the system by discrete event

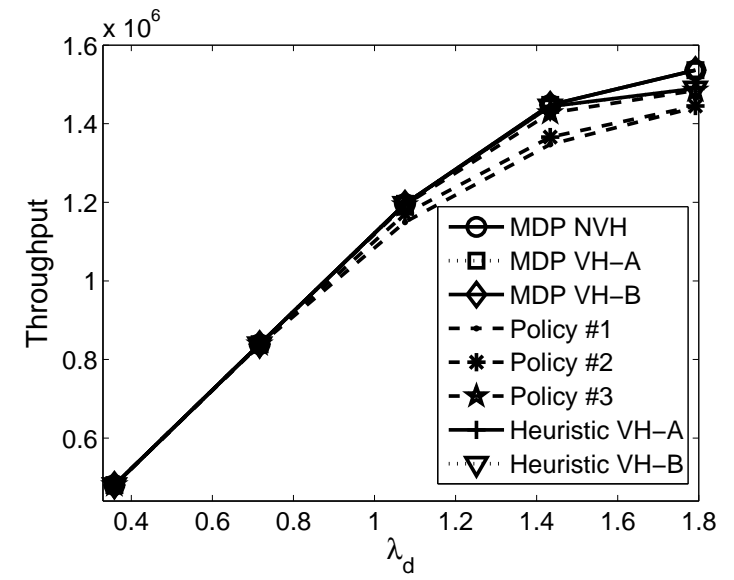

Figure 22: Throughput for various $\lambda_{d}$.

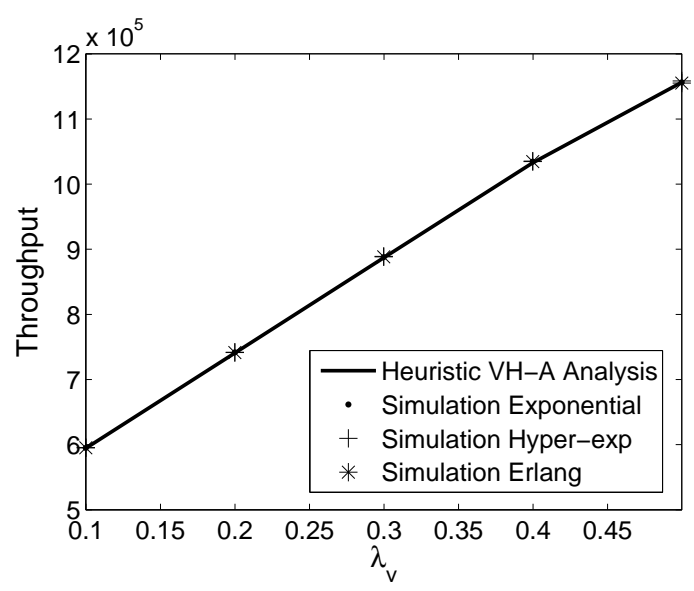

Figure 23: Simulation vs Analysis results of throughput as $\lambda_{v}$ varies.

simulation using three different distributions for the service time of voice calls $T_{v}$ and data size $(\sigma)$, namely, exponential, hyper-exponential and erlang, with variation coefficients of 1,2 and 0.5 respectively. Our purpose here is not only to establish the correctness of our mathematical analysis, but also to asses the impact of the assumption of exponentially distributed data size and voice service time. The results of the simulations for different distributions of data size using heuristic $\mathrm{VH}-\mathrm{A}$ appear on Fig. 23 and Fig. 24. As it is shown, there is an excellent agreement between the analytical model and the simulations. The same applies for the simulations of Heuristic VH-B shown in Fig. 25 and Fig. 26. It is worth noting that all results proved that, under the proposed heuristic policies, the system is insensitive with respect to the distribution of both voice service time and data size beyond their means. 


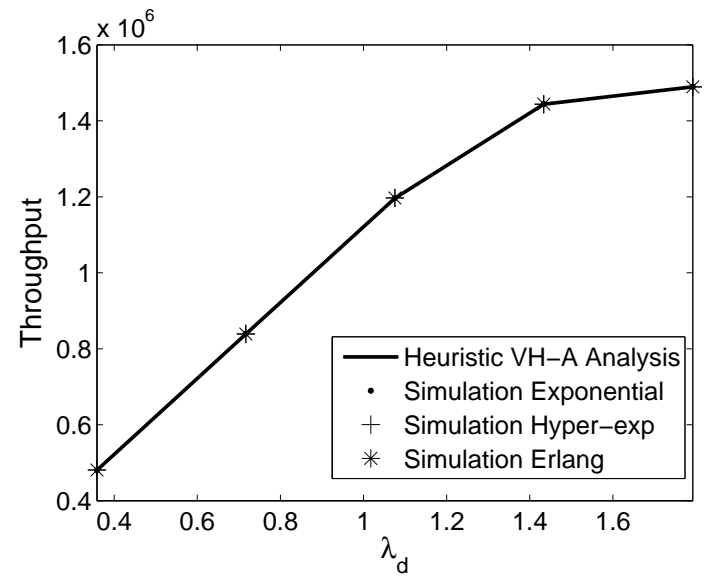

Figure 24: Simulation vs Analysis results of throughput as $\lambda_{d}$ varies.

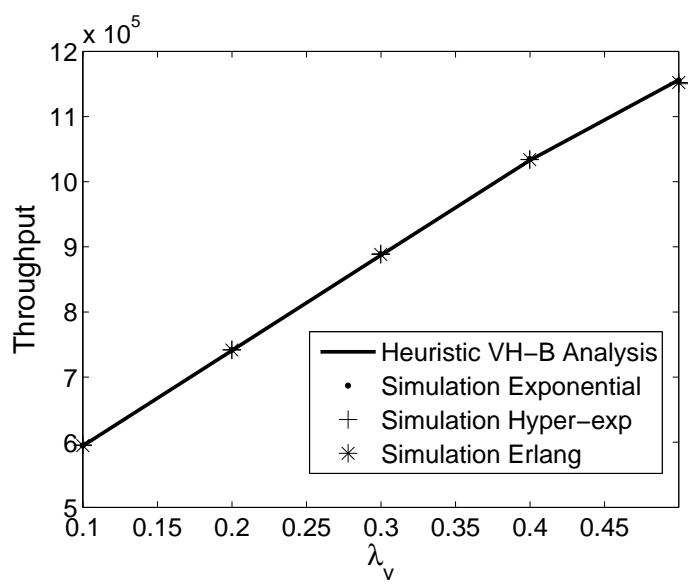

Figure 25: Simulation vs Analysis results of throughput as $\lambda_{v}$ varies.

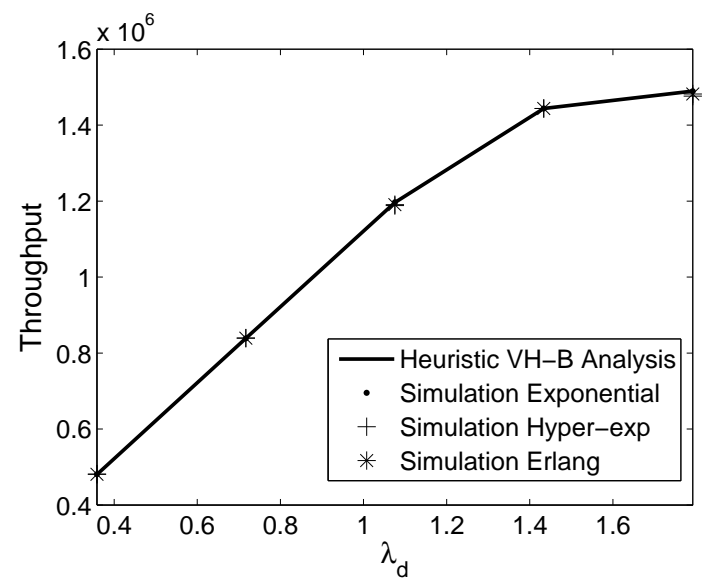

Figure 26: Simulation vs Analysis results of throughput as $\lambda_{d}$ varies.

\section{Cost of the Vertical Handoff}

So far, we have introduced four types of vertical handoff derived from the knowledge we have obtained from the system. These types were used to find new optimal solutions and then two new heuristics were constructed, improving performance over the optimal solutions from the MDP without vertical handoff. The main issue in this section is to explore the impact of adding a cost to vertical handoff.

In order to do this, it is defined a new objective function based on the blocking rates and the vertical handoff rate. The new objective function is defined by

$$
F_{V H}=\theta \cdot \zeta_{V B}+(1-\theta) \cdot \zeta_{D B}+C_{V H} \cdot \zeta_{V H},
$$

where $\theta, 0 \leq \theta \leq 1$, is the factor that defines the cost for blocking a single voice or data call, and $\zeta_{V B}$ and $\zeta_{D B}$ are the mean voice and data blocking rates. In the same way, $C_{V H}$ is the cost of performing a single vertical handoff, and $\zeta_{V H}$ is the mean rate of vertical handoffs performed. Hence, by assigning values to $\theta$ and $C_{V H}$, a new optimal policy that minimizes $F_{V H}$ can be found using a markov decision process.

\subsection{Markov decision process for VH cost}

In this section we define three new MDPs based on (9). The first one does not use vertical handoff (MDP $\mathrm{BR}$ ), the second one uses vertical handoff types I and II (MDP C-1), and the last one uses all four types of vertical handoff (MDP C-2). These MDPs are different from those exposed in Section 7.1 because of their objective functions. The state space for all of them $S$ is defined by (1) and (2) and the set of actions $a$ is that of Table 1 for MDP BR and that of Table 5 for MDP C-1 and MDP C2 . The cost function associated to the objective function for each feasible state $s$ for MDP BR is

$$
\operatorname{cost}(s)=\lambda_{v} \cdot G\left(a_{s}^{v}\right) \cdot \theta+\lambda_{d} \cdot G\left(a_{s}^{d}\right) \cdot(1-\theta),
$$

for MDP $\mathrm{C} 1$ is

$$
\begin{gathered}
\operatorname{cost}(s)=\lambda_{v} \cdot G\left(a_{s}^{v}\right) \cdot \theta+\lambda_{d} \cdot G\left(a_{s}^{d}\right) \cdot(1-\theta) \\
+C_{V H} \cdot\left(\lambda_{v} \cdot R\left(a_{s}^{v}\right)+\lambda_{d} \cdot R\left(a_{s}^{d}\right)\right),
\end{gathered}
$$

and for MDP $\mathrm{C} 2$ is 


$$
\begin{aligned}
\operatorname{cost}(s) & =\lambda_{v} \cdot G\left(a_{s}^{v}\right) \cdot \theta+\lambda_{d} \cdot G\left(a_{s}^{d}\right) \cdot(1-\theta) \\
+ & C_{V H} \cdot\left(\lambda_{v} \cdot R\left(a_{s}^{v}\right)+\lambda_{d} \cdot R\left(a_{s}^{d}\right)\right) \\
+ & C_{V H} \cdot\left(T\left(s_{T D M A}^{v}\right)+T\left(s_{T D M A}^{d}\right)\right. \\
+ & \left.T\left(s_{W C D M A}^{v}\right)+T\left(s_{W C D M A}^{d}\right)\right),
\end{aligned}
$$

where the coefficients are explained in Table 12.

\subsection{Result analysis}

In this section, policy iteration is used to solve the MDPs. The reference scenario is defined in Table 3 with $\theta=0.5$, which means that the cost of blocking voice and data calls is the same.

In Fig. 27 we can see the optimal values for each of the MDPs introduced in the last section for different values of $C_{V H}$. MDP C2 has the lowest optimal value when $C_{V H}=0$ and is followed closely by MDP C1. However, as $C_{V H}$ grows, the value of MDP-R2 rapidly grows surpasing MDP C1 and MDP BR because of the large amount of vertical handoff of types III and IV performed. In fact, for values of $C_{V H}$ as low as 0.01 , the optimal value of MDP C2 is higher than that of MDP BR. This means that when $C_{V H}$ is higher than this, it is not justified to use the policy this MDP uses. Keeping in mind that the cost of blocking voice and data calls is of 0.5 , we could say that it is necesary that the cost of blocking voice and data calls to be about 50 times higher than that of vertical handoff for this policy to be useful.

On the other hand, results found using MDP C1 vary slowly and are limited by those of MDP BR. This occurs because if it is the vertical handoff which causes the objective function to grow, MDP C1 can always choose not to use it, and then its optimal policy will be identical to that of MDP BR. The interesting point here is to find that value of $C_{V H}$ that makes both MDPs to reach the same optimal policiy and therefore the same results. For Fig. 27, when $C_{V H}=0.9$, both policies are the same. This means that when the cost of vertical handoff is about 1.8 times higher than that of blocking voice or data calls, vertical handoff is not useful anymore.

It is interesting to notice that when $C_{V H}=0$, the optimal policies of MDP $\mathrm{C} 1$ and MDP C2 are very similar to those of MDP VH-A and MDP VH-B, even though the objective functions are different. Therefore, the values of parameters such as throughput and blocking probabilities $\left(B P_{v}\right.$ and $\left.B P_{d}\right)$ are similar as well. As $C_{V H}$ grows, the performance of these parameters degrade, and this happens at a faster rate for MDP C2 than for

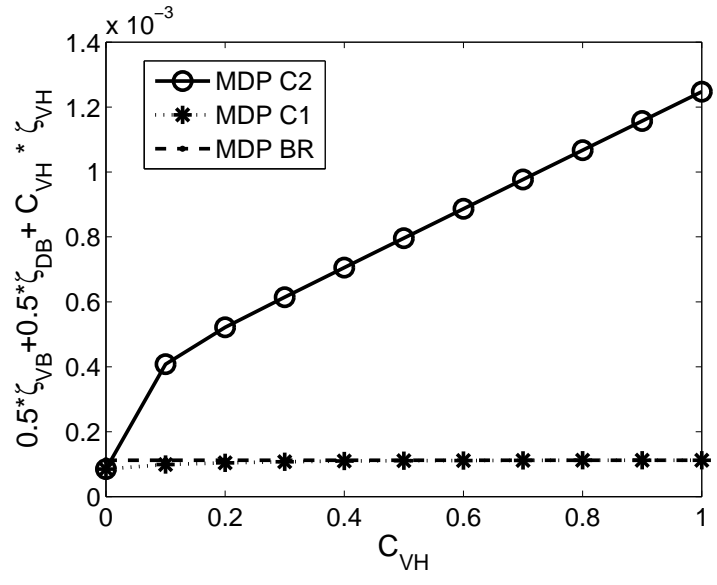

Figure 27: Optimization values for various $C_{V H}$.

MDP C1. Hence, while the ratio of voice/data blocking cost to $C_{V H}$ is high, one could expect that the heuristic policies VH-A and VH-B will fairly represent the optimal policies. This last remark is true even for a higher rank of ratios in the case of MDP $\mathrm{C} 1$ and the heuristic $\mathrm{VH}-\mathrm{A}$, for the reasons explained earlier.

\section{Conclusions}

We have studied the optimal radio access technology selection policy in a system with heterogeneous technologies (WCDMA and TDMA) and services (voice and data) for two different criteria: a function of the blocking probability of each service and the total throughput. The solution method used is policy iteration based on a MDP, which proved to be computationally tractable and robust, since the number of iterations needed is not restrictive, and a solution can be found no matter what initial policy is used. Also, it has been found that one iteration is enough to find a quasioptimal policy, and this can be useful for large systems.

Optimal policies have been found for various traffic scenarios using both optimization functions, and results were compared with those of three fixed policies. Analysis of optimal policies for different scenarios allowed to find the relation in their construction with technologies's inherent characteristics, and also with traffic values and services provided. Based on these characteristics, a heuristic solution has been proposed, and results have shown that it is better than the three fixed policies while data arrival rate is larger than voice arrival rate. Also we have extended the optimization of throughput considering QoS restrictions, and results showed that 


\begin{tabular}{|c|c|c|}
\hline SYMBOL & DEFINITION & VALUE \\
\hline$G\left(a_{s}^{x}\right)$ & $\begin{array}{l}\text { Indicates if } s \text { is a blocking state } \\
\text { for service } x\end{array}$ & $\begin{array}{l}\text { - If action } a_{s}^{x} \text { is blocking, } G\left(a_{s}^{x}\right)=1 \text {. } \\
\text { - Otherwise } G\left(a_{s}^{x}\right)=0 \text {. }\end{array}$ \\
\hline$R\left(a_{s}^{x}\right)$ & $\begin{array}{l}\text { Indicates the number of calls that suffer } \\
\text { vertical handoff when a call of service } x \\
\text { arrives while the system is on state } s \text {. }\end{array}$ & $\begin{array}{l}\text { - If } a_{s}^{x}=3 \text {, and all the conditions for } \\
\text { vertical handoff type I are fulfilled, } \\
R\left(a_{s}^{v}\right)=N \text {. } \\
\text { - If } a_{s}^{x}=4 \text {, and all the conditions for } \\
\text { vertical handoff type II are fulfilled, } \\
R\left(a_{s}^{d}\right)=1 \text {. } \\
\text { - Otherwise, } R\left(a_{s}^{x}\right)=0 \text {. }\end{array}$ \\
\hline$T\left(\boldsymbol{s}_{T D M A}^{v}\right)$ & $\begin{array}{l}\text { Indicates the rate of calls that suffer } \\
\text { vertical handoff when a voice call is } \\
\text { served on TDMA while the system is on } \\
\text { state } s \text {. }\end{array}$ & $\begin{array}{l}\text { - If the conditions for vertical handoff } \\
\text { type IV are fulfilled once a voice call is } \\
\text { served on TDMA, } T\left(s_{T D M A}^{v}\right)=s_{1} \cdot \mu_{v} \text {. } \\
\text { - Otherwise } T\left(s_{T D M A}^{v}\right)=0 \text {. }\end{array}$ \\
\hline$T\left(s_{T D M A}^{d}\right)$ & $\begin{array}{l}\text { Indicates the rate of calls that suffer } \\
\text { vertical handoff when a data call is } \\
\text { served on TDMA while the system is on } \\
\text { state } s \text {. }\end{array}$ & $\begin{array}{l}\text { - If the conditions for vertical handoff } \\
\text { type IV are fulfilled once a data call is } \\
\text { served on TDMA, } \\
T\left(s_{T D M A}^{d}\right)=\min \left(C-s_{1}, s_{2}\right) \cdot B R_{t, d} / \sigma \text {. } \\
\text { - Otherwise } T\left(s_{T D M A}^{d}\right)=0 \text {. }\end{array}$ \\
\hline$T\left(\boldsymbol{s}_{W C D M A}^{v}\right)$ & $\begin{array}{l}\text { Indicates the rate of calls that suffer } \\
\text { vertical handoff when a voice call is } \\
\text { served on WCDMA while the system is } \\
\text { on state } s \text {. }\end{array}$ & $\begin{array}{l}- \text { If the conditions for vertical handoff } \\
\text { type III are fulfilled once a voice call } \\
\text { is served on WCDMA, } \\
T\left(s_{W C D M A}^{v}\right)=s_{3} \cdot \mu_{v} . \\
\bullet \text { Otherwise } T\left(s_{W C D M A}^{v}\right)=0 \text {. }\end{array}$ \\
\hline$T\left(\boldsymbol{s}_{W C D M A}^{d}\right)$ & $\begin{array}{l}\text { Indicates the rate of calls that suffer } \\
\text { vertical handoff when a data call is } \\
\text { served on WCDMA while the system is } \\
\text { on state } s \text {. }\end{array}$ & $\begin{array}{l}\text { - If the conditions for vertical handoff } \\
\text { type III are fulfilled once a data call } \\
\text { is served on WCDMA, } \\
T\left(s_{W C D M A}^{d}\right)=s_{4} \cdot B R_{w, d} / \sigma \text {. } \\
\text { - Otherwise } T\left(s_{W C D M A}^{d}\right)=0 \text {. }\end{array}$ \\
\hline
\end{tabular}

Table 12: Coefficients for vertical handoff cost optimization functions.

adding these do not affect the advantages of the heuristic policy over the others.

We introduced different types of vertical handoff based on the knowledge gained about the behaviour of the system. New optimal solutions were found for a system with more capacity, but their calculation time makes them unfeasible for on-line use. Considering this, new easily programmable heuristics that perform in excellent agreement with the optimal solutions were described and evaluated in different scenarios. Additionally, computer simulations were performed in order to validate the mathematical analysis. Moreover, different probability distributions were checked and, under the proposed policies, the system proved to be insensitive with respect to the the distribution of voice service time and data size beyond their means.

Finally, the cost of vertical handoff was studied in relation to those of voice and data blocking, and some interesting remarks were done in order to understand the impact that vertical handoff has.

\section{Acknowledgements}

This work was supported by the Spanish Government, the European Commission and Universidad Politécnica de Valencia through projects TIN201021378-C02-02, TIN2008-06739-C04-02/TSI and PAID-06-09

\section{References}

[1] Leijia Wu and Kumbesan Sandrasegaran,"A survey on Common Radio Resource Management", The 2nd conference on Wireless Broadband and U-Wideband Communications,AusWireless,p. 66,August,2007.

[2] A. Tolli and P. Hakalin and H. Holma, "Performance Evaluation of Common Radio Resource Management", IEEE International Conference on Communications (ICC), p. 3429-3433, Vol 5, April, 2002. 
[3] Oklabisi E. Falowo and H. Anthony Chan,'Joint Call Admission Control Algorithms: Requirements, Approaches, and Design Considerations",Computer Communications,p. 1200-1217, Vol 31, 2008.

[4] A. Pillekeit and F. Derakhshan and E. Jugl and A. MitscheleThiel, "Force-based Load Balancing in Co-located UMTS/GSM Networks",IEEE 60th VTC 2004-Fall,p. 4402-4406,2004.

[5] K. Suleiman and A. Chan and M. Dlodlo, "Load Balancing in the call admission control of heterogeneous wireless networks", International Wireless Communications and Mobile Computing Conference, p. 245-250, July, 2006.

[6] Fei Yu and Vikram Krishnamurthy, "Optimal Joint Session Admission Control in Integrated WLAN and CDMA Cellular Networks with Vertical Handoff', IEEE Transactions on Mobile Computing, p. 126-139, Vol 6, 2007.

[7] D. Karabudak and C. Hung and B. Bing, "A call admission control scheme using genetic algorithms", ACM Symposium on Applied Computing, p. 1151-1158, 2004.

[8] Agusti R. and O. Sallent and J. Perez-Romero and L. Giupponi, "A fuzzy-neural based approach for joint radio resource management in a beyond 3G framework", 1st International Conference on Quality of Service in heterogeneous in Wire/Wireless networks, p 216-224, 2004.

[9] O. Falowo and H. Chan, "Fuzzy Logic Based Call Admission Control for Next Generation Wireless Networks", 3rd International Symposium on Wireless Communication Systems, $\mathrm{p}$ 574-578, 2006.

[10] Xavier Gelabert and Jordi Perez-Romero and Oriol Sallent and Ramon Agusti, "A Markovian Approach to Radio Access Technology Selection in Heterogeneous Multiaccess/Multiservice Wireless Networks", IEEE Transactions on Mobile Computing, number 10, Vol 7, 2008.

[11] Klein S. Gilhousen and Irwin M. Jacobs and Roberto Padovani and Andrew J. Viterbi and Lindsay A. Weaver and Charles E. Wheatley, "On the Capacity of a Cellular CDMA System", IEEE Transactions on Vehicular Technology, number 2, Vol 40, 1991.

[12] Bertsekas,D.P., "Dynamic Programming and Optimal Control", Athena Scientific, 2001.

[13] Van Leeuwaarden, J. and Aalto, S. and Virtamo, J., "Load balancing in cellular networks using first policy iteration", 5th Finnish Teletraffic Seminar, Citeseer, 2002.

[14] Enrique Stevens-Navarro E., Vincent Wong, "Comparison between Vertical Handoff Decision Algorithms for Heterogeneous Wireless Networks". Vehicular Technology Conference. VTC 2006-Spring. IEEE 63rd. 947-951.(2006)

[15] Pramod Goyal, S.K. Saxena, "A Dynamic Decision Model for Vertical Handoffs across Heterogeneous Wireless Networks". Proceedings of World Academy of Science, Engineering and Technology, Vol.31 (July 2008)

[16] Ahmed Hasswa, Nidal Nasser, Hossam Hassanein, "Generic Vertical Handoff Decision Function for Heterogeneous Wireless Networks". 2nd IFIP International Conference on Wireless and Optical Communications Networks.239-243(2005) 